\title{
Dynamics of poly(ethylene oxide) in a blend with poly(methyl methacrylate): A quasielastic neutron scattering and molecular dynamics simulations study
}

\author{
A.-C. Genix ${ }^{1}$ A. Arbe ${ }^{2}$ F. Alvarez, ${ }^{2,3}$ J. Colmenero, ${ }^{1,2,3, *}$ L. Willner, ${ }^{4}$ and D. Richter ${ }^{4}$ \\ ${ }^{1}$ Donostia International Physics Center, Paseo Manuel de Lardizabal 4, 20018 San Sebastián, Spain \\ ${ }^{2}$ Unidad Física de Materiales, CSIC-UPV/EHU, Apartado 1072, 20080 San Sebastián, Spain \\ ${ }^{3}$ Departamento de Física de Materiales, UPV/EHU, Apartado 1072, 20080 San Sebastián, Spain \\ ${ }^{4}$ Institut für Festkörperforschung, Forschungszentrum Jülich GmbH, D-52425 Jülich, Germany \\ (Received 16 March 2005; revised manuscript received 20 June 2005; published 21 September 2005)
}

\begin{abstract}
In this paper, we have addressed the question of the dynamic miscibility in a blend characterized by very different glass-transition temperatures, $T_{g}$, for the components: poly(ethylene oxide) and poly(methyl methacrylate) (PEO/PMMA). The combination of quasielastic neutron scattering with isotopic labeling and fully atomistic molecular dynamics simulations has allowed us to selectively investigate the dynamics of the two components in the picosecond-10 nanoseconds scale at temperatures close and above the $T_{g}$ of the blend. The main focus was on the PEO component, i.e., that of the lowest $T_{g}$, but first we have characterized the dynamics of the other component in the blend and of the pure PEO homopolymer as reference. In the region investigated, the dynamics of PMMA in the blend is strongly affected by the $\alpha$-methyl rotation; an additional process detected in the experimental window $65 \mathrm{~K}$ above the blend- $T_{g}$ can be identified as the merged $\alpha \beta$ process of this component that shows strong deviations from Gaussian behavior. On the other hand, pure PEO displays entropy driven dynamics up to very large momentum transfers. Such kind of motion seems to freeze when the PEO chains are in the blend. There, we have directly observed a very heterogeneous and moreover confined dynamics for the PEO component. The presence of the hardly moving PMMA matrix leads to the creation of little pockets of mobility where PEO can move. The characteristic size of such confined islands of mobility might be estimated to be of $\approx 1 \mathrm{~nm}$. These findings are corroborated by the simulation study, which has been an essential support and guide in our data analysis procedure.
\end{abstract}

DOI: 10.1103/PhysRevE.72.031808

PACS number(s): 83.80.Tc, 61.12.Ex, 83.10.Rs, 61.25.Hq

\section{INTRODUCTION}

One of the fundamental questions of all polymer based soft matter systems is the way how local friction arises in crowded environments that are chemically heterogeneous. This problem of dynamic miscibility has attracted significant scientific interest recently and experimental information is emerging on a variety of systems [1-21]. Polymers are characterized by a rich multitude of dynamic processes that may extend over more than 15 decades in time or frequency involving various length scales. Apart from vibrations and librational modes, in general polymers display very localized and fast side-group motions (e.g., methyl-group rotations), localized motions involving the secondary $\beta$ relaxation and the diffusive segmental motions which are involved in the glass transition ( $\alpha$ relaxation). It is generally found that the faster and the more localized the molecular motion takes place the less it is affected by blending. For example, it was recently shown by quasielastic neutron scattering (QENS) that the methyl-group rotation in poly(vinyl methyl ether)/ polystyrene (PVME/PS) is hardly sensitive to blending [22]. Similar observations were also made for the dielectric $\beta$ relaxation of PVME in the same system [9]. On the other hand, the structural relaxation, as well as the so-called terminal relaxations, are strongly affected by blending (see, e.g., Refs. $[9,19,20])$.

\footnotetext{
*Electronic address: wapcolej@sc.ehu.es
}

Spectroscopic techniques have usually been employed in order to study these segmental relaxation processes. These experiments resulted in the observation of local heterogeneities. For instance, different magnitudes and temperature dependencies for the dynamics of the different components, different distribution functions and even the observation that one component could exhibit two component relaxation distributions. These observations are very often rationalized in terms of thermally driven concentration fluctuations with spatial regions rich in the component $A$ or $B$ giving rise to environments of different compositions which in turn lead to different glass transition behavior. In all these pictures the length scale of the relevant volume is of key importance. The first model in this direction was proposed by Fischer et al. $[23,24]$, who started from the Donth model of the glass transition [25-27], where a cooperative length scale $\xi \propto(T$ $\left.-T_{0}\right)^{-2 / 3}$ governs the glass transition $\left(T_{0}\right.$, Vogel-Fulcher temperature). The probability for a given concentration fluctuation thereby is related to the structure factor, $S(Q)$. An interpretation of the dielectric spectra in terms of this model as a function of temperature and composition leads to a relevant length scale in the order of $10 \mathrm{~nm}$ close to the glasstransition temperature, $T_{g}$, while the Donth model would require length scales in the order of $2 \mathrm{~nm}$. Kumar et al. [28] extended this model in so far as they introduced the notion that the cooperative volume itself should depend on the distance from the local Vogel-Fulcher temperature, $T-T_{0}$, that corresponds to a given local concentration, i.e., a characteristic volume rich in the lower $T_{g}$ component has a larger 
TABLE I. Number-averaged molecular weight, polydispersity, and cross sections of the samples investigated.

\begin{tabular}{cccccc}
\hline \hline Polymer & $M_{n}(\mathrm{~kg} / \mathrm{mol})$ & $M_{n} / M_{w}$ & $\sigma_{\text {inc }}($ barns $/$ atom $)$ & $\sigma_{\text {coh }}($ barns $/$ atom $)$ & $\sigma_{\text {coh }} / \sigma_{\text {inc }}$ \\
\hline hPEO & 24 & 1.03 & 45.86 & 3.19 & 0.0696 \\
dPEO & 28.3 & 1.07 & 1.17 & 5.39 & 3.607 \\
hPMMA & 25 & 1.1 & 42.81 & 35 & 0.0783 \\
dPMMA & 25.8 & 1.04 & 1.09 & 4.40 & 4.954 \\
hPEO/dPMMA & & & 31.30 & 3.91 \\
dPEO/hPMMA & & & 0.356 & 0.125 \\
\hline \hline
\end{tabular}

distance to $T_{0}$ and therefore is smaller. Again, characteristic lengths in the order of $10 \mathrm{~nm}$ result from an interpretation in terms of this model. Finally, Lodge and McLeish [29] proposed a very simple Ansatz, stating that the relevant length scale for the dynamics in a polymer blend is the Kuhn length of the respective components. No further assumptions on cooperativity effects were made. Local heterogeneities arise from the self-concentration which comes about from the fact that within the volume of a Kuhn length the monomers of a given chain have a higher concentration than on average. With this model McLeish and Lodge were able to describe semiquantitatively the mean component dynamics of a number of miscible polymer blends systems.

In this work, we address the dynamics of the miscible blend of poly(ethylene oxide) and poly(methyl methacrylate) (PEO/PMMA) by quasielastic neutron scattering (NS). PEO/ PMMA is a very unusual blend system as it is characterized by a very large difference in the component glass-transition temperatures, $T_{g}^{\mathrm{PMMA}}=400 \mathrm{~K}, T_{g}^{\mathrm{PEO}} \approx 200 \mathrm{~K}[30,31]$. This system has been studied recently by ${ }^{1} \mathrm{H}$ NMR revealing the very unusual result that, at $20 \%$ blending with PEO at the glass transition of the blend, the PEO segmental dynamics may be up to 9 orders of magnitude faster than that of PMMA [7]. A recent ${ }^{2} \mathrm{H}$ NMR study [20] showed that at lower PEO concentration (3\%) this frequency difference increases to even 12 orders of magnitude. The recent studies also found that the PEO dynamics is relatively independent of the PEO concentration and that the relaxation function is very strongly stretched $(\beta \approx 0.3)$. Finally, Sakai et al. [21] very recently presented quasielastic neutron scattering results on a blend of deuterated PEO and protonated PMMA at $20 \%$ PEO volume fraction addressing thereby the PMMA dynamics. They found a shift of time scale in the PMMA dynamics, which corresponds to the shift of the average glass transition temperature in the blend.

In our work, the main emphasis is directed towards the investigation of the dynamics of the fast PEO component in this blend. It was investigated on a material where the PEO fraction was protonated and the PMMA was deuterated. For reference, also pure PEO as well as the PMMA dynamics in the blend in a sample with deuterated PEO and protonated PMMA were studied. These neutron experiments were complemented by molecular dynamics (MD) simulations that are of invaluable importance for the interpretation of the neutron spectra. We found a significant slowing down of the PEO dynamics in the blend compared to the pure material. We furthermore observed that the fast PEO dynamics in the blend is confined in space to a volume of about $1 \mathrm{~nm}$. This conclusion was reached on the basis of the momentum transfer dependence of the quasielastic spectra. The observation was corroborated by a real space analysis of the MD simulations leading to similar results. The evaluation of the spectral distributions of relaxation times showed that a description in terms of a stretched exponential is not adequate and needs to be replaced by log-normal distribution functions. Again, a detailed comparison with simulations leads to a consistent interpretation. Finally, experiments on the PMMA component in the blend revealed a very important spectral contribution from the $\alpha$-methyl group rotation and showed first PMMA backbone dynamics at $65 \mathrm{~K}$ above the blend glass transition temperature, again in good agreement with the MD simulations.

The paper is organized as follows: (i) First, we describe sample preparation, experimental techniques as well as the simulation approach. (ii) Then, we present selected experimental and simulation results; (iii) thereafter these results are analyzed and brought together; and (iv) finally, the outcome of the analysis is discussed and summarized.

\section{EXPERIMENT}

\section{A. Samples}

The two poly(methyl methacrylate) samples, hPMMA and dPMMA (h, protonated; d, deuterated), were purchased from Polymer Source Inc., Montreal, Cd. The tacticity of the two samples was specified to be $78 \%$ syndiotactic, $2 \%$ isotactic, and $20 \%$ atactic.

The poly(ethylene oxide) polymers, hPEO and dPEO, were prepared by anionic polymerization in tetrahydrofuran (THF) at $50^{\circ} \mathrm{C}$ for 4 days under high vacuum conditions. $\mathrm{h}_{4} \mathrm{EO}$ and $\mathrm{d}_{4} \mathrm{EO}$ (CDN-isotopes, Quebec, Cd, 99.8\% D) were purified twice with calciumhydride, THF with potassium/ benzophenone. As initiator, potassium tert-butoxylate was used for $\mathrm{d}_{4} \mathrm{EO}$ and potassium $n$-hexoxylate for $\mathrm{h}_{4} \mathrm{EO}$. The polymerization was terminated with acetic acid. The polymers were precipitated in cyclohexane and dried under vacuum. Thereafter they were characterized by size exclusion chromatography (SEC) with THF/dimethylacetamide, 90/10 vol/vol, as eluant. The molar masses and polydispersities of all the polymers are given in Table I.

For the scattering experiments, three samples were prepared in rectangular aluminium cells by solvent casting from toluene. The first sample was a blend consisting of $25 \%$ 
$(w / w)$ hPEO in APMMA. The second sample was the inversely labeled blend dPEO 25\% in hPMMA and the third sample was pure hPEO. For each sample a thickness was adjusted according to a calculated transmission value of $90 \%$.

\section{B. Neutron scattering}

The neutron intensity scattered into a solid angle between $\Omega$ and $\Omega+d \Omega$, after having exchanged an energy between $E$ and $E+d E$ with the sample has an incoherent and a coherent contribution [32-35]. The first one can be expressed in terms of incoherent scattering functions $S_{\text {inc }}^{\alpha}(Q, \omega)$ related to the different kinds of nuclei $\alpha(\alpha, \mathrm{H}, \mathrm{C}, \mathrm{O}, \mathrm{D}, \ldots)$ in the sample weighted by their corresponding incoherent cross sections $\sigma_{\text {inc }}^{\alpha} Q Q$ is the modulus of the momentum transfer and $\omega$ $=E / \hbar$. For small energy transfers, $Q=4 \pi \sin (\theta / 2) / \lambda$, where $\theta$ is the scattering angle and $\lambda$ the wavelength of the incoming neutrons. $S_{\text {inc }}^{\alpha}(Q, \omega)$ is the Fourier transform of the intermediate incoherent scattering function $S_{\text {inc }}^{\alpha}(Q, t)$ and the double Fourier transform of $S_{\text {inc }}^{\alpha}(Q, \omega)$ yields the self-part of the van Hove correlation function, $G_{\text {self }}^{\alpha}(r, t)$,

$$
G_{\mathrm{self}}^{\alpha}(r, t)=\frac{1}{N_{\alpha}}\left\langle\sum_{i \alpha=1}^{N_{\alpha}} \delta\left(r-\left|\vec{r}_{i \alpha}(t)-\vec{r}_{i \alpha}(0)\right|\right)\right\rangle,
$$

where $N_{\alpha}$ is the number of nuclei of kind $\alpha$. In the classical limit, it is the probability of a given nucleus of kind $\alpha$ to be at distance $r$ from the position where it was located at a time $t$ before $\left(\vec{r}_{i \alpha}\right.$ denotes the position of the atom $i$ of kind $\alpha$ and the angle brackets mean thermal average). Thus, incoherent scattering looks at correlations between the positions of the same nucleus at different times [32-35].

The coherent scattering deals with relative positions of atomic pairs, i.e., collective dynamics,

$$
\begin{aligned}
& \left(\frac{\partial^{2} \sigma}{\partial \Omega \partial E}\right)_{\mathrm{coh}} \\
& \quad \propto\left\langle\sum_{\alpha, \beta} \sum_{i \alpha, j \beta}^{N_{\alpha}, N_{\beta}} b_{\mathrm{coh}}^{\alpha} b_{\mathrm{coh}}^{\beta} \frac{1}{2 \pi \hbar} \int_{-\infty}^{\infty} e^{-i \omega t} e^{i \vec{Q} \cdot\left[\vec{r}_{i \alpha}(t)-\vec{r}_{j \beta}(0)\right]} d t\right\rangle .
\end{aligned}
$$

Here, $b_{\text {coh }}^{\alpha(\beta)}$ is the scattering length of isotopes of kind $\alpha(\beta)$. For $t=0$ the incoherent contribution is $Q$ independent and given by $\sigma_{\text {inc }} /(4 \pi)\left(\sigma_{\text {inc }}=\Sigma_{\alpha} N_{\alpha} \sigma_{\text {inc }}^{\alpha} / \Sigma_{\alpha} N_{\alpha}\right)$ and the coherent contribution reveals the corresponding partial static structure factor, with an asymptotic value for $Q \rightarrow \infty$ determined by $\sigma_{\text {coh }}=\Sigma_{\alpha} N_{\alpha} \sigma_{\text {coh }}^{\alpha} / \Sigma_{\alpha} N_{\alpha}$, where $\sigma_{\text {coh }}^{\alpha}=4 \pi\left(b_{\mathrm{coh}}^{\alpha}\right)^{2}$.

We note that, as NS spectrometers offer a limited energy resolution, the measured functions are affected by the instrumental resolution function, $R(Q, \omega) . R(Q, \omega)$ is the obtained spectrum when purely elastic $(\hbar \omega=0)$ scattering events take place in the sample [i.e., it is the "image" of $\delta(\omega)]$. It can usually be determined from the scattering of the sample at very low temperature, where all the dynamical processes are frozen. Then, the experimentally accessed quantity is

$$
\frac{\partial^{2} \sigma}{\partial \Omega \partial E} \propto I(Q, \omega) \otimes R(Q, \omega),
$$

where $I(Q, \omega)$ in general contains incoherent and coherent scattering contributions,

$$
I(Q, \omega)=\sigma_{\mathrm{inc}} S_{\mathrm{inc}}(Q, \omega)+4 \pi\left(\frac{\partial \sigma}{\partial \Omega}\right) \tilde{S}_{\mathrm{coh}}(Q, \omega) .
$$

$S_{\text {inc }}(Q, \omega)$ is the averaged incoherent scattering function and $\widetilde{S}_{\text {coh }}(Q, \omega)$ is the normalized coherent scattering function $($ area $=1)$.

In the case of protonated polymers, the scattered intensity is generally dominated by the incoherent contributions from the hydrogens in the sample. This is due to the high value of $\sigma_{\text {inc }}^{\mathrm{H}}$ (80.27 barns) as compared to the other cross sections, $\sigma_{\text {inc }}^{\mathrm{C}, \mathrm{O}}=0, \sigma_{\text {coh }}^{\mathrm{H}, \mathrm{C}, \mathrm{O}}<5.6$ barns. An essential element of NS on soft matter problems is the huge difference between the cross sections of hydrogen and deuterium $\left(\sigma_{\text {inc }}^{\mathrm{D}}=2.05\right.$ barns, $\sigma_{\text {coh }}^{\mathrm{D}}$ $=5.592$ barns), that allows masking the contribution of some molecular groups or components of the system and following the isolated signal of hydrogens in the sample. For this reason, we have used in this work blends of protonated and deuterated chains in order to investigate the dynamics of the hydrogens in the protonated component (see corresponding cross sections in Table I). However, in such mixtures of protonated and deuterated components an important coherent contribution appears at low- $Q$ values due to the contrast between the labeled macromolecules and the matrix, revealing the form factor of the chains.

\section{Diffraction with polarization analysis}

Experimentally it is possible to separate the coherent and incoherent contributions to the scattering by using a spin polarized neutron beam and polarization analysis (see, e.g., Refs. [33,35]). For this purpose, in this work we used the diffuse scattering spectrometer DNS at Jülich, in its diffraction mode [36]. $\lambda$ was set to $3.3 \AA$ enabling a range of scattering vectors $Q$ up to $3.34 \AA^{-1}$. The experiments were performed at $300 \mathrm{~K}$ on both blends and also on the fully deuterated and fully protonated PMMA. The correction for background scattering was done by the measurement of the scattering from the empty cell.

\section{Backscattering}

In a backscattering instrument, the energy resolution is optimized by using single crystal diffraction of cold neutrons with Bragg angles close to $180^{\circ}$ for monochromatization and energy analysis [37]. In this work, we used the backscattering instrument BSS also at Jülich [36]. The energy window of the experiment was set to $-17 \mu \mathrm{eV} \leqslant \hbar \omega \leqslant 17 \mu \mathrm{eV}$. Using $\lambda=6.271 \AA$, we accessed 10 different $Q$ values in the range between $0.16 \AA^{-1}$ and $1.87 \AA^{-1}$. The energy resolution was $\delta E(\mathrm{HWHM}) \approx 0.45 \mu \mathrm{eV}$ for the detectors corresponding to $Q>0.6 \AA^{-1}$ and slightly worse for the smaller scattering angles $\left(0.7 \mu \mathrm{eV}\right.$ for $\left.0.16 \AA^{-1}\right)$. The samples were positioned at $45^{\circ}$ with respect to the incident beam. The temperatures investigated were 350,375 , and $400 \mathrm{~K}$ for 
hPEO, from 300 to $400 \mathrm{~K}$ each $25 \mathrm{~K}$ for the hPEO/dPMMA blend and 350 and $400 \mathrm{~K}$ for the dPEO/hPMMA blend. Measuring times were of the order of $20 \mathrm{~h}$. The experimental resolution function was obtained from measurements on each sample at $5 \mathrm{~K}$. Background corrections were performed by subtracting the scattering of the empty cell. The efficiency of the detectors was taken into account by dividing the background corrected spectra by the integrals of the spectra obtained from a highly incoherently scattering sample at $4 \mathrm{~K}$.

\section{MD SIMULATIONS}

The simulations were carried out by using the Insight (Insight II 4.0.0 P version) and the Discover-3 module from Molecular Simulations Inc. (now Accelrys) with the Polymer Consortium Force Field (PCFF) (detailed information can be found in Refs. [38-41]). The model system was built by means of the well-known Amorphous Cell protocol $[42,43]$. In this work, a cubic cell containing one polymer chain of 23 monomer units of PEO and four identical polymer chains of 25 monomer units each of PMMA with a tacticity degree close to $80 \%$ syndiotactic was constructed at $580 \mathrm{~K}$ in a cell under periodic boundary conditions, with a density fixed to be unity. Such a density leads to a cell dimension of $26.36 \AA$ of side. The chemically realistic simulations we are using are limited to rather small simulation boxes if we want to be able to follow the model system over a time range of a few nanoseconds. The system constructed corresponds to a blend of PEO/PMMA with a composition in weight of about $10 \%$ of PEO. We have chosen this low-PEO concentration in order to be sure that no clusterization of PEO chains occurs. In any case, as it was mentioned in the Introduction, the experimental results available suggest that the PEO dynamics in the blends hardly depends on the concentration when this is lower than 30\% [20]. Therefore, the simulated results are expected to be comparable to those obtained by NS on the $25 \%$ PEO-content sample, at least at a semiquantitative level.

Standard minimization procedures (Polak-Ribiere conjugate gradients method) were applied to the constructed sample in order to minimize the so obtained energy structure, and subsequent dynamics was run for $1 \mathrm{~ns}$ in order to equilibrate the sample. The chosen temperature is high enough to allow local structural equilibration of the sample in this time. The system obtained in this way was used as a starting point for collecting data every $0.01 \mathrm{ps}$ during a MD run of $1 \mathrm{~ns}$. As integration method we have used the velocity-Verlet algorithm with a time step of $1 \mathrm{fs}$. The simulations were carried out in the NVT ensemble (that is by means of keeping constant the number of atoms, the cell size and the temperature of the sample). However, instead of a real temperature-bath coupling (Nose-Hoover or Berendsen thermostats, for instance) in order to control the temperature, we have followed a rather crude velocity scaling procedure but allowing for a wide temperature window of $10 \mathrm{~K}$, within which no rescaling is applied. Under these conditions, greater temperature fluctuations are allowed but the trajectory is less disturbed.
In fact, in a previous work [44] we have checked that by following this simple procedure we obtain results similar to those obtained with a $N V E$ ensemble (by keeping constant the sample total energy instead of its temperature), which has the proper Newtonian dynamics. After the first collecting data $1 \mathrm{~ns}$ MD run, two more successive runs of 2 and $20 \mathrm{~ns}$ were carried out, collecting data every 0.05 and 0.5 ps, respectively. Nearly indistinguishable results were obtained from the different simulation runs. Thus, no signature of any aging process was observed during the successive runs, confirming local equilibration of the sample. Subsequently, the constraint previously imposed by choosing the density to be unity was now released by means of tuning its value through a $1 \mathrm{~ns}$ long run under NPT conditions, that is by fixing in this case the value of the pressure (chosen to be the atmospheric one $P=0.0001 \mathrm{GPa}$ ) and allowing the cell size to rearrange to the new conditions (that is letting the density rearrange since the number of atoms is constant). All this was done by means of the Berendsen procedure. The temperature during this $1 \mathrm{~ns}$ run was decreased from the initial value of $580 \mathrm{~K}$ to a final value of $400 \mathrm{~K}$ and the final cell so obtained was found to correspond to a density of $1.080 \mathrm{~g} / \mathrm{cm}^{3}$ with a cell dimension of $25.70 \AA$ of side. At this new temperature the same procedure as before was followed, i.e., during a first 1 ns run the new cell was allowed to equilibrate. Afterwards, a $1 \mathrm{~ns}$ run was run collecting data every $0.01 \mathrm{ps}$. A subsequent 2 ns run was found to be in agreement with this one, and, finally, a 20 ns long run was launched, collecting data every 0.5 ps. Most of the results reported here correspond to the simulation runs at $400 \mathrm{~K}$.

\section{RESULTS}

\section{A. Static structure factor}

Figure 1 displays an overview of the results obtained for the structure factors of pure PMMA and PEO or PMMA, respectively, in the blend. The data were obtained by polarization analysis and reflect the coherent scattering from the respective samples. The structure factor $S(Q)$ from pure dPMMA [Fig. 1(a)] is characterized by three significant maxima in the $Q$ interval between 0 and $3 \AA^{-1}$, distinguishing this material from other polymers, where normally only two peaks are observed. While the high $Q$ peak at $Q$ $\approx 3 \AA^{-1}$ is characteristic for many polymers and reflects local intrachain correlations [45], the two other peaks relate to the polymer packing in the melt. In particular, the low- $Q$ maximum at about $Q \approx 0.9 \AA^{-1}$ signifies a rather larger interchain distance of about $d \approx 2 \pi / Q=7 \AA$. This low- $Q$ peak appears again in the hPEO/dPMMA material as the shoulder of the form factor forward scattering [Fig. 1(b)], while it is absent in the dPEO/hPMMA material [Fig. 1(c)]. This absence is corroborated by the data from pure hPMMA, where again a low- $Q$ peak is missing. The main structure factor peak in dPEO/hPMMA at around $Q=1.3 \AA^{-1}$ probably reflects contributions from PEO correlations, which first peak is also located at this $Q$-value [46], as well as from the carbons of PMMA-we note that for the protonated sample $S(Q)$ also shows there its main peak [see Fig. 1(a)]. 


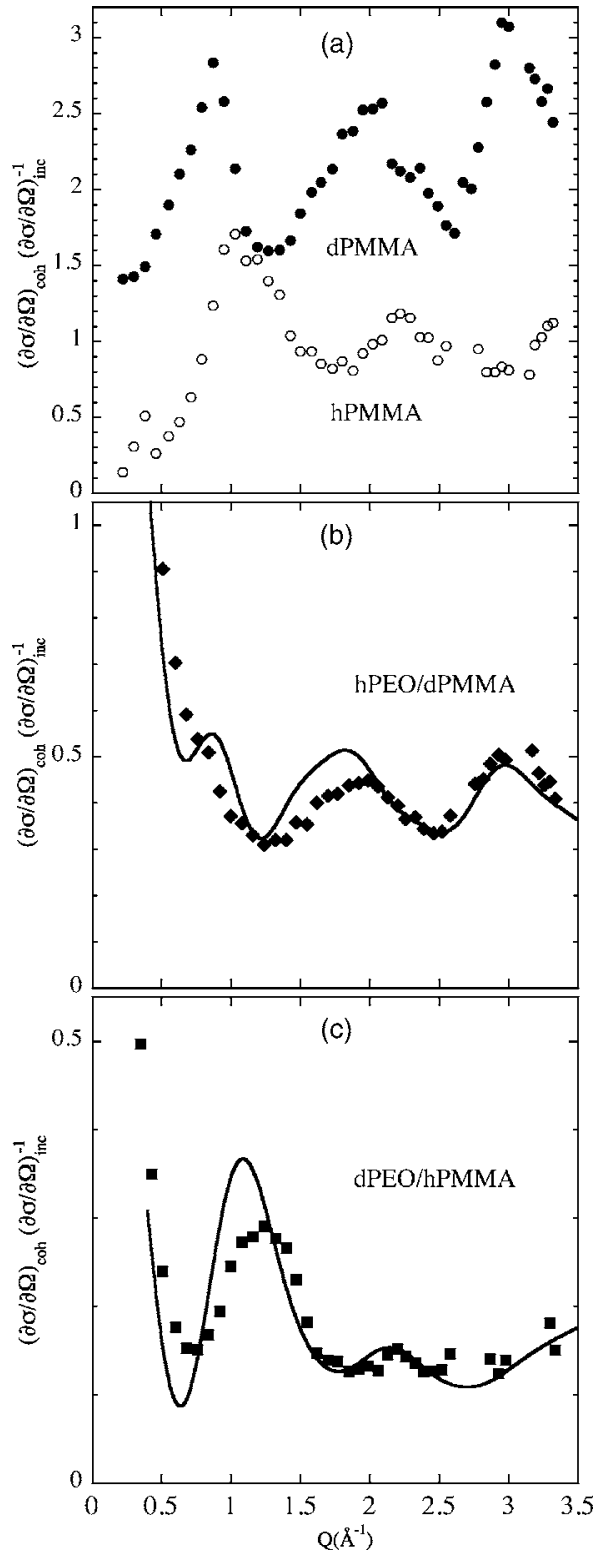

FIG. 1. Ratio between the coherent and incoherent scattering intensities measured by means of DNS at $300 \mathrm{~K}$ (symbols) and calculated from MD simulations at $400 \mathrm{~K}$ (continuous lines). (a) Fully deuterated PMMA (full circles) and fully protonated PMMA (multiplied by 10) (empty circles). (b) Blend where PEO is protonated and PMMA deuterated. (c) Blend where PEO is deuterated and PMMA is protonated. The simulation data have been multiplied by 0.455 in (b) and 2 in (c).

We further remark that the strong increase of the scattering at low $Q$ in the blend materials reflects the polymer form factor. Finally, we note that in the PEO protonated blend material the fraction of coherent scattering is quite significant (see Table I). In the $Q$ range $Q \geqslant 1 \AA^{-1}$, it amounts to $30 \%-50 \%$ of the incoherent scattering and thus contributes significantly to the total scattering of this sample. On the other hand, for the PMMA protonated sample the respective coherent scattering fraction is much weaker (except for small angles).

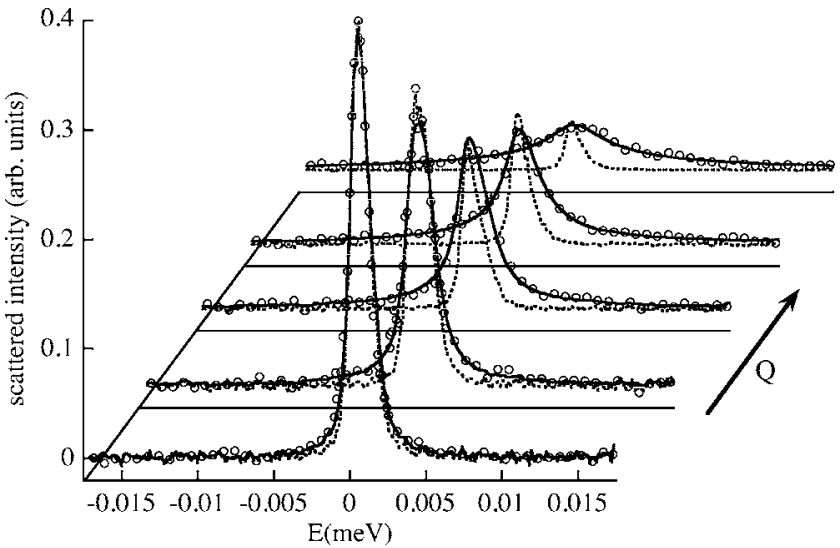

FIG. 2. Neutron scattering spectra of pure PEO at $350 \mathrm{~K}$ and different $Q$ values, $0.16,0.24,0.32,0.41$, and $0.56 \AA^{-1}$, from front to back. The dotted lines show the instrumental resolutions obtained at $5 \mathrm{~K}$; the solid lines are fits to the Fourier transform of KWW functions with $\beta=0.5$.

\section{B. Backscattering}

\section{Pure PEO}

Figure 2 displays spectra obtained from the pure PEO sample in the low- $Q$ regime $0.16 \AA^{-1} \leqslant Q \leqslant 0.56 \AA^{-1}$ at the backscattering spectrometer at $350 \mathrm{~K}$. The dotted lines represent the resolution function of the instrument. From Fig. 2 the very strong dispersion of the quasielastic broadening in these spectra is evident. While for $Q=0.16 \AA^{-1}$ only very weak quasielastic wings may be seen, at $Q=0.56 \AA^{-1}$ we observe a very much broadened spectrum, indicating a quasielastic width which increases with a strong function of $Q$.

\section{PEO in the blend}

As mentioned already in Sec. IV A in this sample not only the incoherent scattering contribution from the PEO but also the coherent scattering from the PMMA fraction is significant. Therefore, we should comment briefly on the normalization of the data. All spectra were here normalized to a purely incoherent scatterer measured at $T=4 \mathrm{~K}$. Figure 3 compares the outcome of the normalization of the lowtemperature backscattering data from the blend sample with the measured $Q$-dependent intensity obtained from the diffuse scattering experiment [Fig. 1(b)]. In order to compare we have added both the coherent and the incoherent contributions from the DNS experiment. Figure 3 shows that a very consistent normalization of the data could be obtained.

Keeping in mind that the measured intensities from this sample combine contributions from the mobile PEO component and the very much less mobile PMMA component, Fig. 4 displays the QENS results for this blend at $350 \mathrm{~K}$ and compares them with the spectra observed for pure PEO. While at small $Q\left(Q=0.56 \AA^{-1}\right)$ hardly any broadening of the PEO in the blend spectrum is visible, at the larger $Q$ $=1 \AA^{-1}$ the observed spectrum reveals itself as a combination of an elastic peak-same width as the resolution function-and a broad quasielastic spectrum (dashed line). Evidently the dynamics of PEO in the blend is very strongly 


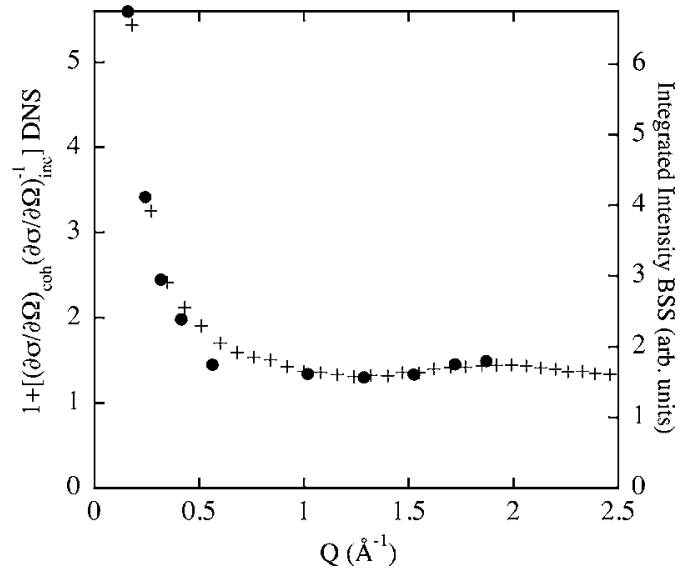

FIG. 3. Momentum transfer dependence of the total intensity scattered by the hPEO/dPMMA sample, the integrated intensities of the $5 \mathrm{~K}$ BSS spectra (full circles) are compared with the addition of the incoherent and coherent intensities measured by DNS at $300 \mathrm{~K}$ (plus signs).

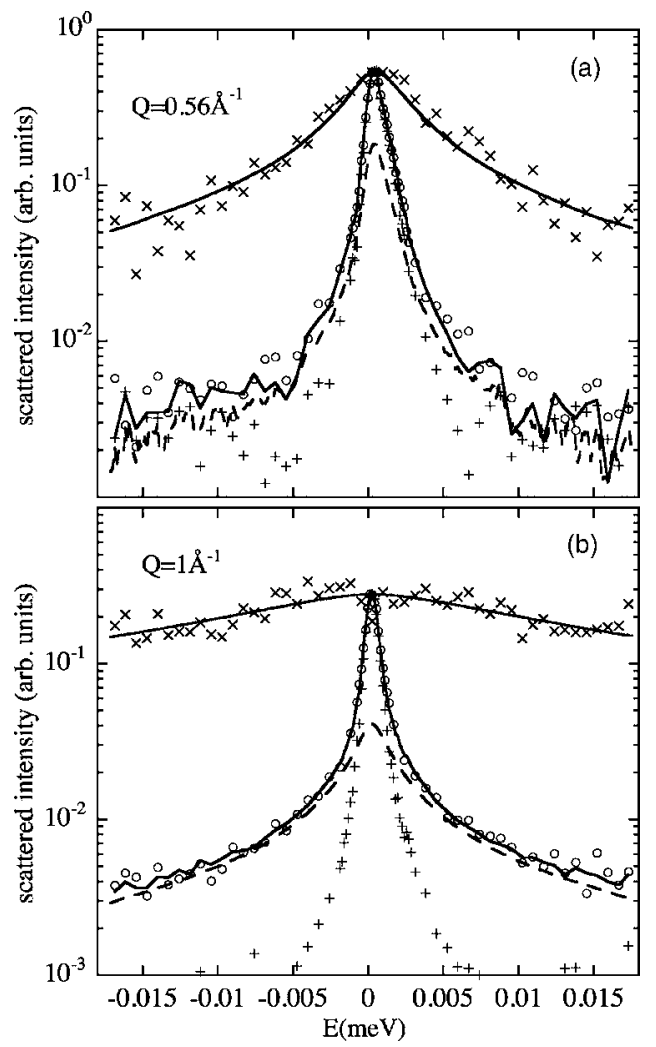

FIG. 4. Comparison of neutron scattering spectra obtained for pure PEO (crosses) and PEO in the blend (circles) at $350 \mathrm{~K}$ and at (a) $Q=0.56 \AA^{-1}$ and (b) $Q=1.02 \AA^{-1}$. Pure PEO data are normalized to the maximum of the blend peak. The plus signs show the instrumental resolution obtained at $5 \mathrm{~K}$. For pure PEO, the solid lines are fits to the Fourier transform of KWW functions $(\beta=0.5)$ (see Sec. V B 1). For hPEO/dPMMA, the dashed lines are fits of the quasielastic contribution of the PEO hydrogens to the Fourier transform of KWW functions $(\beta=0.41)$, and the solid lines are the sum of an elastic component and the dashed quasielastic component (see Sec. V B 3).

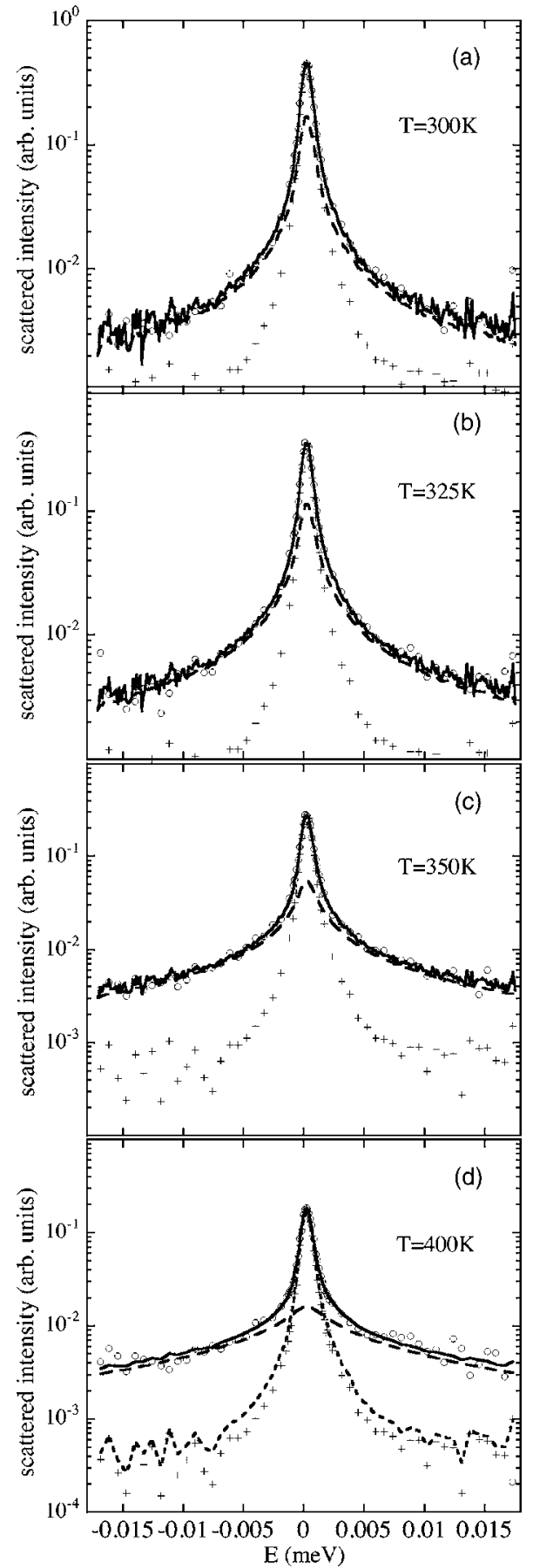

FIG. 5. Effect of temperature on QENS spectra of hPEO/ dPMMA at $Q=1.02 \AA^{-1}$, (a) $300 \mathrm{~K}$, (b) $325 \mathrm{~K}$, (c) $350 \mathrm{~K}$, and (d) $400 \mathrm{~K}$. The plus signs show the instrumental resolution obtained at $5 \mathrm{~K}$. The dashed lines are fitting curves using a log-normal distribution of relaxation times for the fast component corresponding to PEO dynamics. The solid lines show the sum of such quasielastic component and the coherent contribution, which in (a)-(c) is elastic and in (d) shows a broadening depicted by the dotted line (Fourier transform of a KWW with $\beta=0.5$, see Sec. V B 3).

slowed down compared to the pure material (we remind that the broadening of the spectra with respect to the resolution function is a measure of the inverse of the average relaxation time). 
Figure 5 displays the spectrum at $Q=1 \AA^{-1}$ for four different temperatures. In each case a well-defined narrow component superimposed on a broad quasielastic spectrum is visible. This quasielastic spectrum broadens significantly with increasing temperature and finally at $T=400 \mathrm{~K}$ most of the quasielastic intensity is outside the BSS dynamic window.

\section{PMMA in the blend}

As demonstrated in Fig. 1(c) for the dPEO/hPMMA sample, the incoherent scattering from PMMA strongly prevails-only around $Q=1 \AA^{-1}$ a coherent contribution from dPEO is expected. Figure 6 presents results for two different $Q$ values, $Q=1.3 \AA^{-1}$ and $Q=1.9 \AA^{-1}$, at $350 \mathrm{~K}$ and $400 \mathrm{~K}$. Again the spectra are characterized by a superposition of a narrow contribution and broad quasielastic wings which become more pronounced with increasing $Q$ and temperature.

\section{MD simulations}

From the atomic trajectories obtained in the simulation runs and taking into account the different neutron scattering lengths for the different atoms, we have calculated the structure factors [Eq. (2)] for the two blend samples which were studied experimentally, hPEO/dPMMA and $\mathrm{dPEO} / \mathrm{hPMMA}$. The obtained results are presented in the form of the ratio between coherent and incoherent intensities in Fig. 1 in comparison with the experimental results. As the composition of the simulated and real blend samples is not the same, a comparison of the coherent intensities measured and simulated cannot be done in absolute units. Therefore, we have multiplied the simulated results by the appropriate factors $(0.455$ for hPEO/dPMMA and 2 for $\mathrm{dPEO} / \mathrm{hPMMA}$ ), in order to match the high- $Q$ range of the experimental curves. After this procedure, we obtain a good agreement between simulation and experimental results, in particular, taking into account that the composition and the temperature are not the same. This means that the packing of PEO chains within the PMMA matrix is well reproduced by the simulated cell. Also the low- $Q$ features revealing the form factor of polymer chains are nicely reproduced by the simulated data although the accessible $Q$-range is limited in this case to $Q$ $\geqslant 0.4 \AA^{-1}$ due to the size of the simulated cell.

From the atomic trajectories obtained in the simulations we have also calculated the self-part of the van Hove correlation function, $G_{\text {self }}(r, t)$, [see Eq. (1)] for the different atoms. Since we want to connect with neutron scattering results, we will focus on the results obtained for different hydrogen atoms in the samples. Figure 7 shows the corresponding mean squared displacements, calculated as the second moment of $G_{\text {self }}(r, t)$,

$$
\left\langle r^{2}(t)\right\rangle=\int_{0}^{\infty} r^{2} 4 \pi r^{2} G_{\text {self }}(r, t) d r .
$$

The figure shows separately $\left\langle r^{2}(t)\right\rangle$ corresponding to different hydrogen atoms contained in a protonated blend, PEO hydrogens; main-chain hydrogens of PMMA; ester-methyl group hydrogens of PMMA; $\alpha$-methyl hydrogens of PMMA

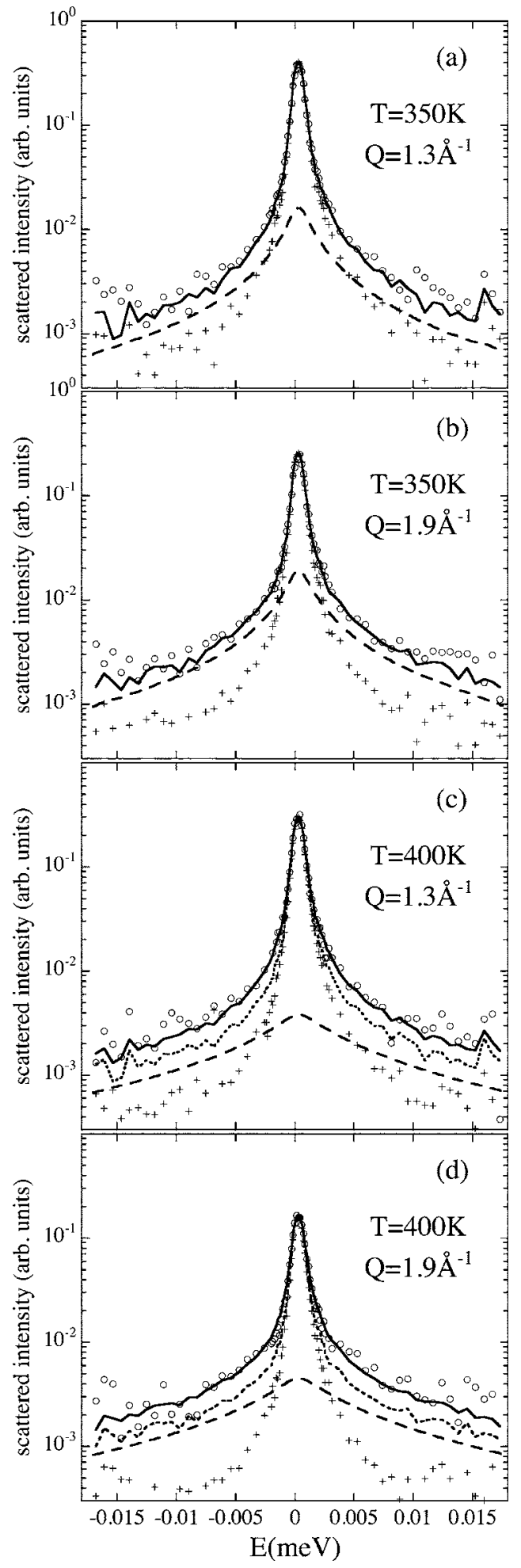

FIG. 6. Neutron scattering spectra of dPEO/hPMMA at $350 \mathrm{~K}$ at (a) $Q=1.29 \AA^{-1}$, (b) $Q=1.87 \AA^{-1}$ and at $400 \mathrm{~K}$ at (c) $Q$ $=1.29 \AA^{-1}$, (d) $Q=1.87 \AA^{-1}$. The plus signs show the instrumental resolution obtained at $5 \mathrm{~K}$. At $350 \mathrm{~K}$, the solid lines are fits by means of $\alpha$-methyl rotation. At $400 \mathrm{~K}$, the dotted lines show the effective resolution giving account for the $\alpha$-methyl rotation (see Sec. V B 2) and the solid lines are fits to the Fourier transform of KWW functions $(\beta=0.5)$. The dashed lines show the quasielastic components corresponding to methyl-group dynamics. 


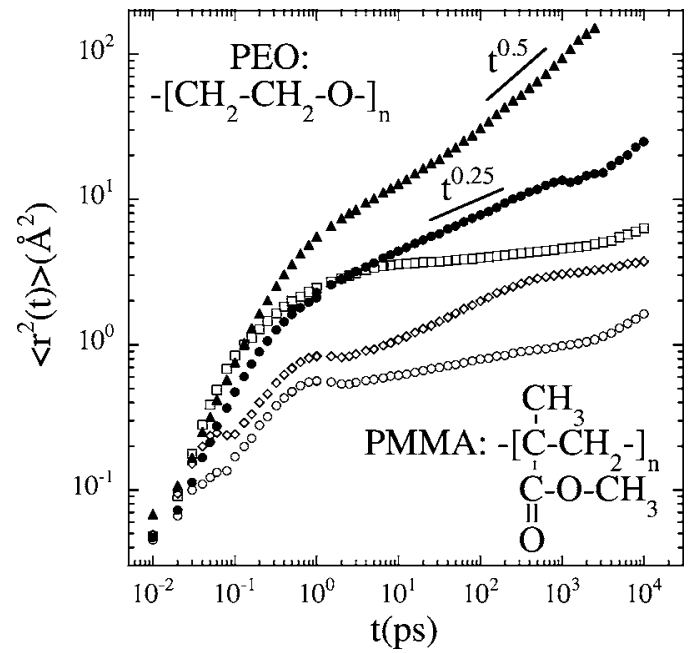

FIG. 7. Mean squared displacement for different hydrogen atoms of the simulated blend calculated from the MD trajectories at $400 \mathrm{~K}$, PEO hydrogens (full circles); main-chain hydrogens of PMMA (empty circles), ester-methyl group hydrogens of PMMA (squares); $\alpha$-methyl group hydrogens of PMMA (diamonds). Results corresponding to PEO hydrogens at $580 \mathrm{~K}$ are also shown for comparison (full triangles).

(see chemical formulas in the figure). These results correspond to $400 \mathrm{~K}$. Moreover, the values of $\left\langle r^{2}(t)\right\rangle$ obtained for PEO hydrogens at a high temperature of $580 \mathrm{~K}$ are also shown for comparison. In this latter case, the behavior of $\left\langle r^{2}(t)\right\rangle$ is what is expected for a glass-forming polymer above the glass transition, after the microscopic regime until $\approx 1 \mathrm{ps}$, there is a "decaging" region which crosses over to a subdiffusionlike behavior $\left\langle r^{2}(t)\right\rangle \propto t^{0.5}$ [47]. However, at 400 $\mathrm{K}$ the $\left\langle r^{2}(t)\right\rangle$ corresponding to PEO hydrogens apparently does not show any decaging regime. A very weak time dependence $\left\langle r^{2}(t)\right\rangle \propto t^{0.25}$ extends from 1 ps to longer times. Only at about $1 \mathrm{~ns}$ there is a signature of some kind of saturation (localization), which seems to break again at the limit of our simulation time. We have checked that this effect is repetitive at different simulation runs and is not an artifact of poor statistics.

On the other hand, the results from the PMMA hydrogens at $400 \mathrm{~K}$ do not show any clear indication of a structural relaxation. The mean squared displacement of PMMA mainchain hydrogens only displays a very weak time dependence after the microscopic regime and only at about $2 \mathrm{~ns}$ there seems to be an indication of decaging. The same behavior is displayed by the ester-methyl group hydrogens, indicating that the ester-methyl group rotation takes place at this temperature at very short times, below $10 \mathrm{ps}$. The activation energy for this methyl group is very low (about $60 \mathrm{meV}$ ) [48] and therefore it can be expected that the dynamics associated with its rotation has characteristic times of the order of some picosecond at $400 \mathrm{~K}$. The $\left\langle r^{2}(t)\right\rangle$ from the $\alpha$-methyl group hydrogens nicely shows the step corresponding to the methyl-group rotation taking place in the time range from $\approx 2$ ps to $\approx 2 \mathrm{~ns}$. This qualitative analysis suggests that, at least until about 2-3 ns, the PEO is moving within a more or less rigid matrix of PMMA, which mainly shows very localized motions as methyl-group rotations.

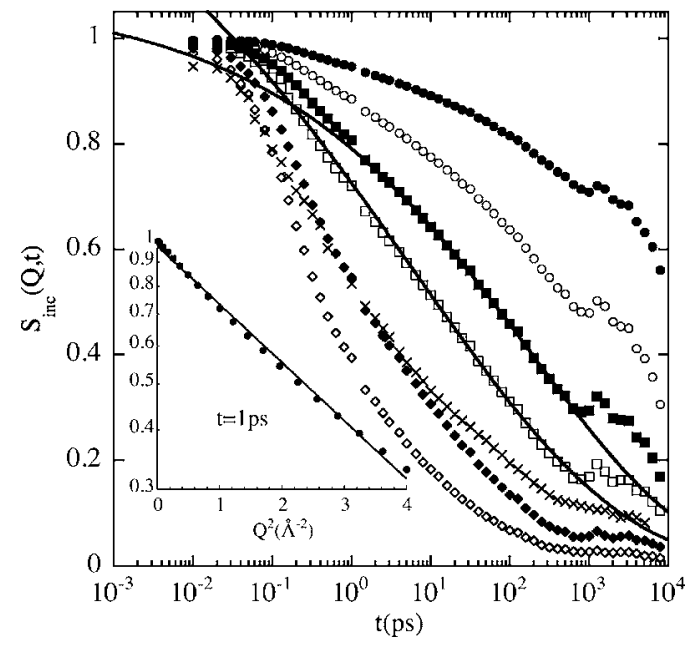

FIG. 8. Intermediate incoherent scattering function $S_{\text {inc }}(Q, t)$ calculated from the MD trajectories for the hydrogen atoms of PEO in the simulated blend at $400 \mathrm{~K}$. Different curves correspond to different $Q$ values, $0.4 \AA^{-1}$ (full circles), $0.6 \AA^{-1}$ (empty circles), $0.8 \AA^{-1}$ (full squares), $1.0 \AA^{-1}$ (empty squares), $1.4 \AA^{-1}$ (full diamonds), and $1.8 \AA^{-1}$ (empty diamonds). The correlation function followed by NMR is also displayed (crosses). The solid lines show the result of fits of KWW functions to the decay at $0.8 \AA^{-1}(\beta$ $=0.22)$ and at $1.0 \AA^{-1}(\beta=0.17)$ between 5 and 1000 ps. The inset shows the value of $S_{\text {inc }}(Q, t)$ at $t=1$ ps for different $Q$ 's showing a typical DWF behavior.

By Fourier transforming the calculated $G_{\text {self }}(r, t)$ the intermediate scattering functions $S_{\text {inc }}(Q, t)$ in $Q$ space have been obtained. Figure 8 shows $S_{\text {inc }}(Q, t)$ calculated for the hydrogens of PEO in the blend at $400 \mathrm{~K}$ for several $Q$ values. As usually found in glass-forming systems above $T_{g}$, the decay becomes more pronounced with increasing $Q$. However, what is clearly different from the typical behavior observed is that apparently there is no clear separation of the so-called microscopic dynamics from the slower decay attributed to the structural relaxation. Normally, this is manifested as a kink in $S_{\text {inc }}(Q, t)$ at about 2 ps reflecting the cage effect (see, e.g., Ref. [44]). The correlations here decay in a rather continuous way leading to strongly stretched spectra. We note also the presence of a kind of plateau in the curves in the nanosecond region before the function definitely decays to zero, a feature reflecting the saturation of the $\left\langle r^{2}(t)\right\rangle-$ also absent in homopolymers. In this figure we have also displayed the autocorrelation function for the C-D bond vector which is observed by NMR [20]. It almost coincides with $S_{\text {inc }}\left(Q=1.4 \AA^{-1}, t\right)$, though showing an even stronger stretching and a more marked plateau.

Moving to PMMA in the blend, Fig. 9 displays the results for $S_{\text {inc }}(Q, t)$ calculated for the different types of hydrogens at $400 \mathrm{~K}$ and different $Q$ values. The main-chain hydrogens in Fig. 9(a) show a first fast decay in the microscopic region, below $\approx 1 \mathrm{ps}$, followed by a very broad and slow decay. A qualitatively similar behavior is observed for the estermethyl group hydrogens [Fig. 9(b)]; in this case, however, the fast decay extends towards longer times, $\approx 10 \mathrm{ps}$ instead of $\approx 1 \mathrm{ps}$, and leads to more pronounced decays for all $Q$ values. This is because it is partially produced by the rota- 


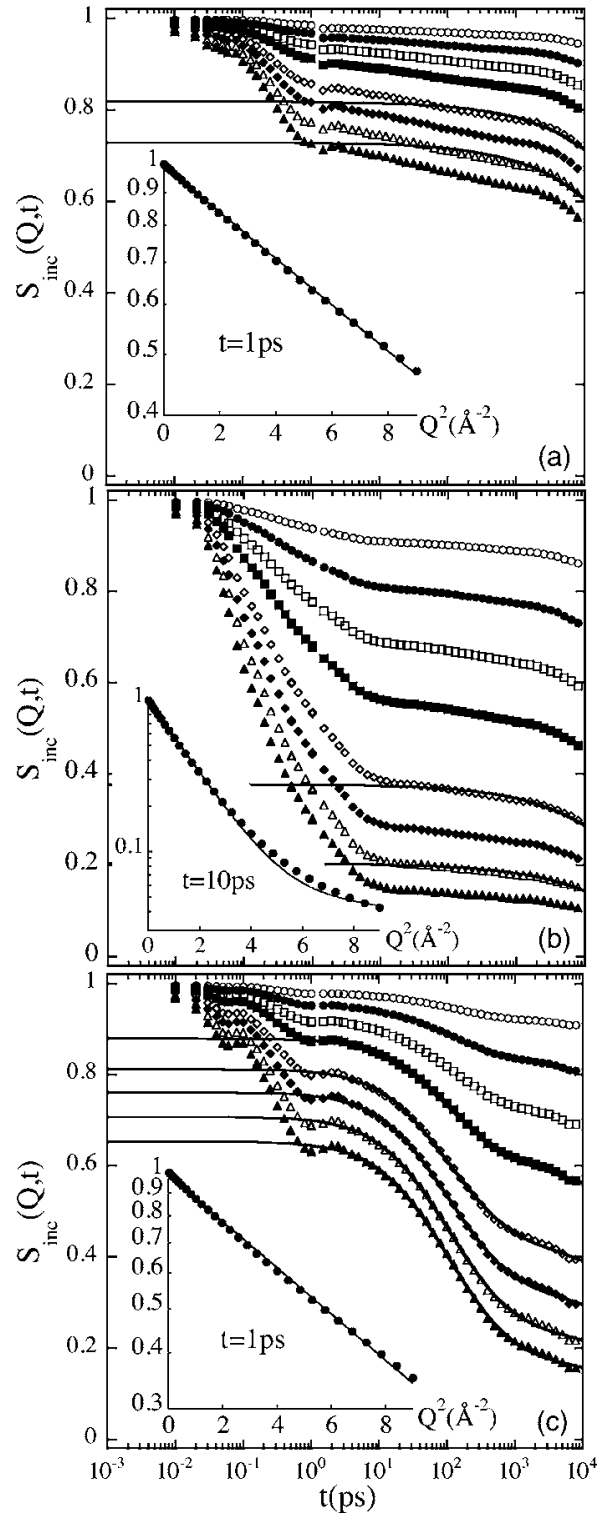

FIG. 9. Intermediate incoherent scattering function, $S_{\text {inc }}(Q, t)$, calculated from the MD trajectories for the different hydrogen atoms of PMMA in the simulated blend, main-chain hydrogens (a), ester-methyl group hydrogens (b), and $\alpha$-methyl group hydrogens (c). In the three cases the different curves correspond to different $Q$ values, $0.4,0.6,0.8,1.0,1.3,1.5,1.7$, and $1.9 \AA^{-1}$ (top to bottom). The solid lines are fits with KWW functions $(\beta=0.5)$ of the decays above $10 \mathrm{ps}$ in (a) and (b), and with the combination of methyl rotation and KWW $(\beta=0.5)$ of the decays above $5 \mathrm{ps}$ in (c). Insets, $Q$ dependence of the values of these functions at $t=1 \mathrm{ps}, S_{\text {inc }}(Q, t$ $=1 \mathrm{ps}$ ) (a) and (c) and at $t=10 \mathrm{ps}, S_{\text {inc }}(Q, t=10 \mathrm{ps)}$ (b). The lines are descriptions in terms of a DWF in (a) and (c) and the product of a DWF and the elastic incoherent structure factor $I_{E}(Q)$ of a methylgroup rotation in (b).

tional motion of the ester-methyl group. Finally, for the $\alpha$-methyl group hydrogens a clear three-step decay is observed for $S_{\text {inc }}(Q, t)$ [Fig. 9(c)]. After the first fast process below $\approx 1 \mathrm{ps}$, rather similar to that displayed by the backbone hydrogens, a marked additional decay is observed in the time range between $\approx 2 \mathrm{ps}-1 \mathrm{~ns}$. This is a signature of

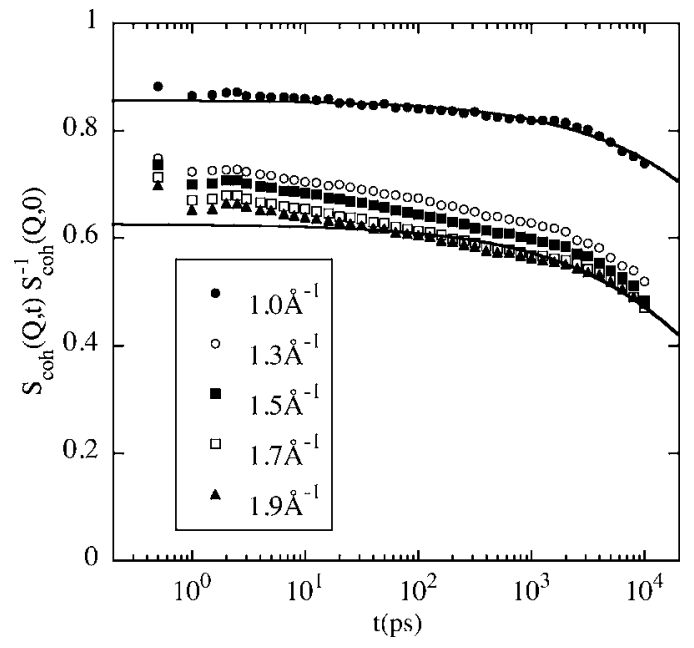

FIG. 10. Coherent intensity corresponding to the hPEO/ dPMMA simulated sample at $400 \mathrm{~K}$ as calculated from the MD trajectories. Different curves correspond to the different $Q$ values indicated. All the curves are normalized to the values at $t=0$ (static case). The solid lines are fits with KWW functions $(\beta=0.5)$ of the decays above 10 ps for two selected $Q$ values $\left(1\right.$ and $\left.1.9 \AA^{-1}\right)$.

the rotation of this group, that is more hindered than the ester-methyl. We note that the time scale where this process takes place, some hundreds of picoseconds, is relevant for BSS. A third regime evident after $\approx 1$ ns leads to a further slow decrease of the correlation function.

Last, in order to facilitate the analysis of the experimental data corresponding to the hPEO/dPMMA sample, we have also computed the coherent scattering function (dynamic structure factor) corresponding to this system at $400 \mathrm{~K}$. This was evaluated from the atomic trajectories following the socalled "Filon" method [49], which is a well-known and established procedure. As we have seen, the coherent contribution there is not negligible and impossible to be directly accessed from an experimental point of view. The corresponding results are presented in Fig. 10 for selected $Q$ values in the relevant range for the experimental data. In the BSS window, this function is not completely flat at $400 \mathrm{~K}$ and shows a very stretched behavior.

\section{ANALYSIS}

\section{A. Computer simulations}

We will start with the analysis of the simulations results because they will be used as a qualitative guide to analyze neutron scattering data. We will focus our analysis on the time range above some picoseconds, which is relevant for the structural relaxation in glass-forming systems. We note that the BSS window is centered approximately in the 0.04-6 ns region and is sensitive mainly to the dynamics taking place in this interval.

The usual description of the slow decay $(t>2-5 \mathrm{ps})$ of the intermediate scattering function of the atoms in a glassforming polymer is performed in terms of a stretched exponential or Kohlrausch-Williams-Watts (KWW) function, 


$$
S_{\mathrm{inc}}^{\mathrm{KWW}}(Q, t)=A(Q) \exp \left[-\left(\frac{t}{\tau_{w}}\right)^{\beta}\right] .
$$

Here $\beta$ is the stretching parameter describing the shape of the relaxation function $(0<\beta \leqslant 1)$ and $\tau_{w}$ the relaxation time which may depend on $Q$ and $T$. The amplitude $A(Q)$ is a generalized Debye-Waller factor (DWF) or Lamb-Mössbauer factor (LMF) accounting for the first fast decay of $S_{\text {self }}(Q, t)$,

$$
A(Q)=\exp \left(-\frac{\left\langle u^{2}\right\rangle}{3} Q^{2}\right),
$$

where $\left\langle u^{2}\right\rangle$ is the mean-squared displacement associated to the fast decay step.

It turns out that this description cannot be easily applied in the case of the hydrogens of PEO in the blend. As an example, Fig. 8 shows the fits delivered by Eq. (6) for two $Q$ values. The resulting values for the shape parameter $\beta$ are in the range $0.15-0.3$, very low compared to what is usually found in polymeric systems $(\beta=0.4-0.5)$ (see, e.g., Ref. [50]). In addition, such fits lead to unphysical amplitudes well above 1 , up to 4 , mainly in the high- $Q$ range. In fact, the values of the amplitudes tend to increase with increasing $Q$, which is contrary to the behavior of a real DWF [Eq. (7)]. The proper amplitude could be estimated from, e.g., the value of $S_{\text {inc }}(Q, t)$ at $t=1 \mathrm{ps}$. A Debye-Waller factor taken in such a way yields a value of $\left\langle u^{2}\right\rangle \approx 0.84 \AA^{2}$ (see inset in Fig. 8 ), which is very reasonable for a polymeric system at such high temperature [51-53]. Moreover, we note that the KWW description fails at long times (beyond $\approx 1 \mathrm{~ns}$ ), where a kind of plateau seems to develop. All these findings imply that the response function of PEO in the blend cannot be easily described by a stretched exponential. On the other hand, the observation of a broad response function agrees with the NMR data reported in Ref. [20], which were analyzed in terms of Eq. (6) without knowing the amplitude. Taking into account the simulated data of the C-D reorientation function, which is measured by NMR, one can realize that if one imposes realistic amplitudes on the fit, a limited quality of the KWW description is obtained also for that reorientational function.

We now turn to the results of PMMA in the blend. First, the broad slow decay after $\approx 2$ ps for the backbone hydrogens and after $\approx 10$ ps for the ester-methyl group hydrogens is very stretched-almost logarithmic — until about $1 \mathrm{~ns}$, and followed by a steeper time dependence (see Fig. 9). By restricting the fitting range to $10 \mathrm{ps}-10 \mathrm{~ns}$ for both kinds of atoms, an analysis in terms of KWW functions with a common value of $\beta=0.5$ is possible [see Figs. 9(a) and 9(b)], though the quality of the description is rather limited for the main-chain atoms.

Concerning the hydrogens of the $\alpha$-methyl group, we have seen that after the microscopic dynamics and the methyl-group rotation, $S_{\text {inc }}(Q, t)$ at $400 \mathrm{~K}$ also shows a clear indication of an additional decay-methyl-group rotation should give rise to a constant value at long times, as will be shown below. As the $\alpha$-methyl group is directly linked to the main chain, one can expect that the $\alpha$-methyl hydrogens also participate in the main-chain motions. In this situation, a good approximation [54] is to consider that both kinds of motions are statistically independent, i.e., the $\alpha$-methyl hydrogens simultaneously rotate and participate in the overall segmental dynamics. This translates into a product of the corresponding intermediate scattering functions,

$$
S_{\text {inc }}^{\alpha-\mathrm{MG}}(Q, t)=S_{\mathrm{inc}}^{\mathrm{rot}}(Q, t) S_{\mathrm{inc}}^{\mathrm{seg}}(Q, t) .
$$

The incoherent scattering function for the rotational motion, $S_{\text {inc }}^{\text {rot }}(Q, t)$, can be derived in the following way: Let us assume a hydrogen in a methyl group rotates with a characteristic rate $\Gamma$. $\Gamma$ varies with temperature according to an Arrhenius law,

$$
\Gamma=\Gamma_{\infty} \exp \left(-\frac{E_{A}}{k_{B} T}\right),
$$

where $\Gamma_{\infty}$ is a preexponential factor and $E_{A}$ the activation energy for the rotation. The scattering function for such motion is

$$
S_{\text {inc }}^{\Gamma}(Q, t)=I_{E}(Q)+\left[1-I_{E}(Q)\right] \exp (-\Gamma t),
$$

where the elastic incoherent structure factor $I_{E}(Q)$ (usually called EISF) carries the information about the geometry of the particular motion involved. For a rotational jump between three equivalent positions, it is given by

$$
I_{E}(Q)=\frac{1}{3}\left(1+2 \frac{\sin \left(Q r_{\mathrm{HH}}\right)}{Q r_{\mathrm{HH}}}\right)
$$

$\left(r_{\mathrm{HH}}=1.78 \AA\right.$, distance between the hydrogens). It is well known that the disorder inherent to the amorphous state leads to the appearance of distributions of rates in glassy systems. This ingredient was introduced by the so-called rotation rate distribution model [55,56], which considers as a first approximation a log-Gaussian distribution of hopping rates

$$
H(\log \Gamma)=\frac{1}{\sqrt{2 \pi} \sigma_{\Gamma}} \exp \left(-\frac{\left(\log \Gamma-\log \Gamma_{0}\right)^{2}}{2 \sigma_{\Gamma}^{2}}\right) .
$$

$\sigma_{\Gamma}$ is the width of the distribution centered at $\Gamma_{0}$. Thus, the final scattering function for a hydrogen undergoing rotation in a methyl group in a glass forming system, $S_{\text {inc }}^{\text {rot }}(Q, \omega)$, is built by adding the scattering functions of the hydrogens located in the different environments weighted by the distribution function,

$$
S_{\mathrm{inc}}^{\mathrm{rot}}(Q, t)=\int_{-\infty}^{+\infty} H(\log \Gamma) S_{\mathrm{inc}}^{\Gamma}(Q, t) d(\log \Gamma) .
$$

The incoherent scattering function for segmental motion $S_{\text {inc }}^{\text {seg }}(Q, t)$ has the functional form describing the slow decay of the main-chain hydrogens. We have used a KWW function [Eq. (6)] with $\beta=0.5$. In the range of applicability of this model function (after the fast microscopic decay) the achieved description of the MD-simulation results is excellent, as can be appreciated in Fig. 9(c). From the fit, a value of $\Gamma_{0}=4.45 \mu \mathrm{eV}$ and a width of $\sigma_{\Gamma}=0.58$ decades are found.

Figure 11 compares the KWW characteristic times $\tau_{w}$ obtained for the three atomic species. The $Q$ dependence of $\tau_{w}$ in all cases can be described by a power law $\tau_{w} \propto Q^{-\kappa}$, with values of $\kappa$ in the order of 2 . We have also deduced the 


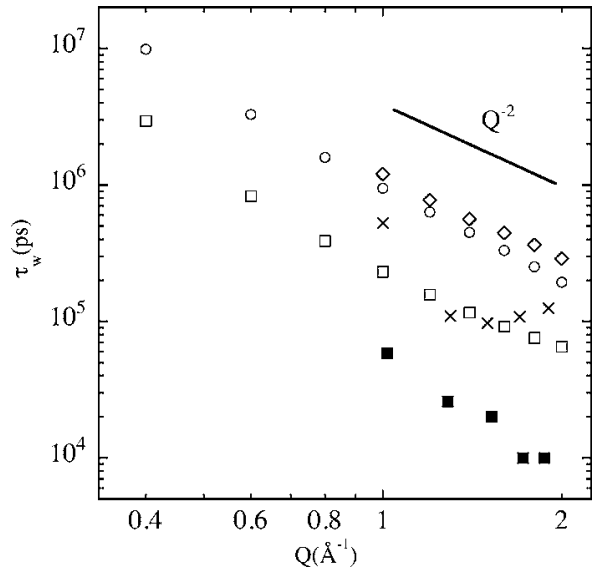

FIG. 11. Momentum transfer dependence of the characteristic times obtained from the fit of the scattering functions calculated from the MD simulations by means of KWW functions with $\beta$ $=0.5$ (see text), incoherent scattering function for main chain (empty circles), ester-methyl (empty squares), and $\alpha$-methyl hydrogens (empty diamonds) of PMMA in the blend and coherent scattering function for the hPEO/dPMMA sample (crosses). The results obtained for the segmental motion of PMMA in the blend from the experimental data of the $\mathrm{dPEO} / \mathrm{hPMMA}$ sample (see text) are displayed as full squares for comparison.

values of $\left\langle u^{2}\right\rangle$ in the DWF from the $Q$ dependence of $S_{\text {inc }}(Q, t)$ after the first decay. For main-chain and $\alpha$-methyl hydrogens we find rather similar values $\left(0.25\right.$ and $0.35 \AA^{2}$, respectively) from $S_{\text {inc }}(Q, t \approx 1 \mathrm{ps})$ (see insets in Fig. 9). In the case of the ester-methyl hydrogens, as the librational dynamics and the rotation are not well separated in time, we have taken the value of the correlation function after the global decay in the microscopic region (10 ps) and fitted to the product of a DWF [Eq. (7)] and the elastic incoherent structure factor $I_{E}(Q)$ [Eq. (11)] for methyl-group rotation. A good description of the data is found [see inset in Fig. 9(b)], corroborating that the decay is due to such a combined dynamics. A higher value of $0.55 \AA^{2}$ for $\left\langle u^{2}\right\rangle$ is obtained.

Finally, in the time range relevant for our BSS experiments it is also possible to describe the coherent scattering function of the hPEO/dPMMA at $400 \mathrm{~K}$ by means of the same functional form [Eq. (6) with $\beta=0.5$ ] (see Fig. 10). The obtained time scales mirror the $Q$ dependence of the static structure factor [see Fig. 1(b)] and are in the same range as the time scales determined from the KWW descriptions of the various self-correlation functions. We note that motions leading to the same initial and final atomic configurations, as the methyl-group rotations, do not contribute to quasielastic coherent scattering. Therefore, an average of the time scales for only segmental motion of the hydrogens of PMMA in the blend can be considered as a good approach to the coherent contribution in the hPEO/dPMMA sample.

\section{B. Neutron scattering}

The neutron scattering spectra were fitted with a numerical convolution of a theoretical function and the instrumental resolution function [see Eq. (3)].

\section{Analysis of pure PEO}

The spectra from pure PEO were described in terms of the Fourier transformed stretched exponential [see Eq. (6)]. Free fits of $\beta$ lead to an average $\beta$ value of 0.44 for the different $Q$ 's and temperatures. This value is close to the expected $\beta$ of the Rouse model $(\beta=0.5)$ [57]. This model treats the dynamics of a Gaussian chain in a heat bath [58]. Thereby, it implicitly assumes that on the considered length and time scales only entropic restoring forces drive the dynamics. $S_{\text {inc }}^{\text {Rouse }}(Q, t)$ is described by a KWW with $\beta=0.5$ and a characteristic time

$$
\tau_{\text {inc }}^{R}=\frac{9 \pi}{W \ell^{4}} Q^{-4}
$$

with $W=3 k_{B} T / \zeta \ell^{2}$ the elementary Rouse frequency. $W$ is the ratio of the entropic force, $3 k_{B} T / \ell^{2}$, and the friction coefficient $\zeta . \ell^{2}$ is the mean-square segment length. We thus fixed $\beta=0.5$ for describing our spectra. As can be seen in Fig. 2 they are well represented by this function. We note that at high $Q$, the fast dynamics of PEO leads to a vanishing intensity in the BSS window and, as a consequence, data at $Q$ $>0.56 \AA^{-1}$ were disregarded. The KWW relaxation time, $\tau_{w}$, and an amplitude factor $[A(Q)$ in Eq. (7)] were varied. Figure 12(a) displays the $Q$ and temperature dependent $\tau_{w}$ times. The strong decrease of $\tau_{w}$ with increasing $Q$ follows very well the Rouse prediction [Eq. (14)] for the three temperatures investigated. From the results obtained for $\tau_{w}$ we can deduce the $T$-dependent Rouse frequencies $W \ell^{4}$ through Eq. (14). They are displayed in Fig. 12(b).

\section{Dynamics of PMMA in the blend}

The spectra from the sample dPEO/hPMMA reveal the dynamics of PMMA in the blend, through the self-motions of its hydrogens. The lowest temperature experimentally investigated, $350 \mathrm{~K}$, is close to the $T_{g}$ of the blend, $\approx 335 \mathrm{~K}$ [7]. Therefore, it is expected that the quasielastic broadening cannot relate to any PMMA backbone motions but only to localized processes. As suggested by the MD simulations at $400 \mathrm{~K}$, these would be the $\alpha$-methyl rotations and then we might write the total incoherent scattering function as

$$
S_{\mathrm{inc}}(Q, \omega)=A(Q)\left[n_{e} I_{E}(Q) \delta(\omega)+n_{\mathrm{MC}} \delta(\omega)+n_{\alpha} S_{\mathrm{inc}}^{\mathrm{rot}}(Q, \omega)\right],
$$

where $n_{e}, n_{\mathrm{MC}}$, and $n_{\alpha}$ are the relative abundances of hydrogens in the ester-methyl, main chain, and $\alpha$-methyl, respectively, and $S_{\text {inc }}^{\text {rot }}(Q, \omega)$ is the Fourier transform of Eq. (13). Here we are assuming that the DWF is the same for all kinds of atoms and the rotations of the ester-methyl are already very fast and therefore, their contribution has decayed down to the elastic level dictated by the $\operatorname{EISF}\left[I_{E}(Q)\right.$ in Eq. (11)]. Apart from the DWF $A(Q)$, the free parameters in this model are $\Gamma_{0}$ and $\sigma_{\Gamma}$. We can even fix the value of $\sigma_{\Gamma}$ by using additional independent information from the librational peak. Existing inelastic measurements on the density of states of PMMA containing a deuterated ester-methyl group $[60,61]$ locate the first librational level of the $\alpha$-methyl at $43 \mathrm{meV}$, which translates into a mean activation energy of $E_{A_{0}}$ 

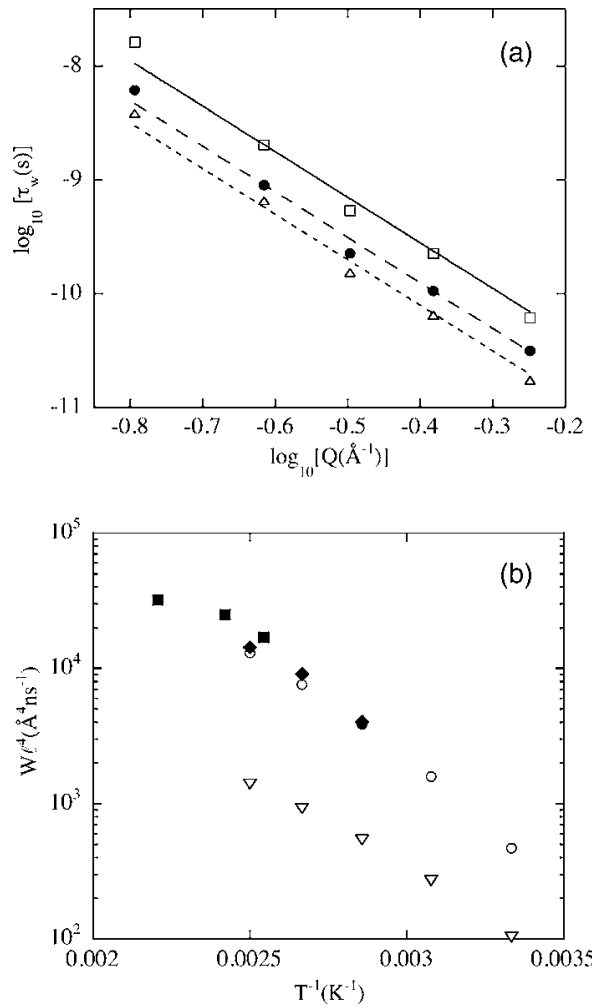

FIG. 12. (a) $Q$ dependence of the characteristic time, $\tau_{w}$, for pure $\mathrm{PEO}$ at $350 \mathrm{~K}$ (squares), $375 \mathrm{~K}$ (circles), and $400 \mathrm{~K}$ (triangles). The lines are fits with a power law $\tau_{w} \propto Q^{-4}$. (b) Temperature dependence of the Rouse rate, $W \ell^{4}$, deduced from this work (full diamonds) and obtained from neutron spin-echo measurements [59] (full squares). For comparison, NMR prediction coming from the literature [Ref. [20] (empty triangles) and Ref. [7] (empty circles)] are also displayed.

$=290 \mathrm{meV}$. The width of this peak [61] reveals a width of $\sigma_{E} \approx 39 \mathrm{meV}$ for the underlying distribution of activation energies, that implies via the relation

$$
\sigma_{\Gamma}=\frac{\sigma_{E} \ln (e)}{k_{B} T}
$$

a value of 0.56 for $\sigma_{\Gamma}(T=350 \mathrm{~K})$. Under these assumptions, the fit of Eq. (15) to the spectra at $350 \mathrm{~K}$ leads to very good agreements, as can be appreciated from Figs. 6(a) and 6(b). The obtained value for $\Gamma_{0}$ is $4.37 \mu \mathrm{eV}$, corresponding to a characteristic time of $\tau_{\text {rot }}=3 /\left(2 \Gamma_{0}\right)=226$ ps.

At $400 \mathrm{~K}$ the description of the spectra by Eq. (15) fails. Using the values determined from Eq. (9) and Eq. (16) for the $\alpha$-methyl rotation corresponding to $400 \mathrm{~K}, \Gamma_{0}(T$ $=400 \mathrm{~K})=14.5 \mu \mathrm{eV}$ and $\sigma_{\Gamma}(T=400 \mathrm{~K})=0.49$, too narrow functions are obtained [see dotted lines in Figs. 6(c) and 6(d)]. Even leaving these parameters floating, a satisfactory fit is not achieved. Thus, the need to introduce an additional dynamical process becomes evident, as it was also found in the simulations at the same temperature. In a parallel way, we have assumed that the additional dynamics can be described by a KWW function in the time domain with a $\beta$ value of 0.5 , and that the $\alpha$-methyl rotation and this slower process are statistically independent [Eq. (8)]. We note that the product in Eq. (8) translates into the convolution of the respective functions in the frequency domain. As experimentally we cannot distinguish the different kinds of hydrogens in our sample, the same time scale and DWF are assumed for all of them. As can be seen in Figs. 6(c) and 6(d), this approach allows to a very good description of the experimental data, and provides the $Q$-dependent characteristic times, $\tau_{w}$, that would be an average of the characteristic times for segmental motion corresponding to the different kinds of hydrogens in PMMA. The obtained values follow a power law in $Q$ with an exponent close to -3 and are plotted in Fig. 11 .

Apart from the interest of the analysis of PMMA dynamics in the blend, we note that these results will be of help for the analysis of the data of the hPEO/dPMMA sample, in particular for the characterization of the coherent contribution. In the low- $T$ regime below and around $350 \mathrm{~K}$, the only active process of PMMA in the BSS window is the $\alpha$-methyl group rotation. As mentioned above, this is not visible for coherent scattering and therefore, the coherent contribution should be elastic in such temperature range. On the other hand, at $400 \mathrm{~K}$ the additional motion observed for PMMA in the blend should lead to quasielastic coherent scattering. This is strongly supported by the MD simulations, that suggests that we could use the KWW average component for segmental dynamics of PMMA as a good approximation for the coherent contribution in the hPEO/dPMMA sample at this high temperature.

\section{PEO in the blend}

In the hPEO/dPMMA sample, the main contribution is coming from hydrogen atoms of the PEO component and is incoherent. From the previous MD-simulation results, we know that the dynamics of PEO in the blend should be faster than the other contributions (dominated by the coherent contribution). Thus, we may define a general expression for the total scattering function as

$$
\begin{aligned}
I(Q, \omega)= & \sigma_{\text {inc }}^{\mathrm{H}, \mathrm{PEO}} S_{\text {inc }}^{\mathrm{H}, \mathrm{PEO}}(Q, \omega) \\
& +\left[\sigma_{\text {inc }}^{\mathrm{D}, \mathrm{PMMA}}+4 \pi\left(\frac{\partial \sigma}{\partial \Omega}\right)_{\text {coh }}\right] \widetilde{S}_{\text {slow }}(Q, \omega) .
\end{aligned}
$$

$S_{\text {inc }}^{\mathrm{H}, \mathrm{PEO}}$ and $\widetilde{S}_{\text {slow }}(Q, \omega)$ are given by the proper DWF $[A(Q)]$ multiplying the respective normalized scattering functions

$$
\begin{gathered}
S_{\text {inc }}^{\mathrm{H}, \mathrm{PEO}}(Q, \omega)=A^{\mathrm{H}, \mathrm{PEO}}(Q) \varphi_{\text {inc }}^{\mathrm{H}, \mathrm{PEO}}(Q, \omega), \\
\tilde{S}_{\text {slow }}(Q, \omega)=A^{\text {slow }}(Q) \varphi^{\text {slow }}(Q, \omega) .
\end{gathered}
$$

Due to the large number of parameters involved-note that the structure factor might also change with temperature-we may assume that both DWF are the same and that the cross sections can be fixed as those determined from DNS at $300 \mathrm{~K}$. In order to account for possible small deviations from these hypotheses we introduced a fitting parameter $f_{\mathrm{PEO}}$ describing the relative amount of the PEO scattering intensity compared to the total intensity, 
TABLE II. Parameters characterizing the dynamics of PEO in the blend. The characteristic times correspond to $Q=1.02 \AA^{-1}$.

\begin{tabular}{ccccc}
\hline \hline Temperature $(\mathrm{K})$ & $\beta$ & $\sigma$ & $\tau_{0}(\mathrm{ps})$ & $\left\langle u^{2}\right\rangle\left(\AA^{2}\right)$ \\
\hline 300 & 0.32 & 1.34 & 323 & $0.23 \pm 0.13$ \\
325 & 0.36 & 1.08 & 175 & $0.14 \pm 0.08$ \\
350 & 0.41 & 0.87 & 38 & $0.32 \pm 0.17$ \\
375 & 0.45 & 0.74 & 40 & $0.44 \pm 0.14$ \\
400 & 0.50 & 0.66 & 24 & $0.66 \pm 0.25$ \\
\hline \hline
\end{tabular}

$$
I(Q, \omega)=A(Q)\left(f_{\mathrm{PEO}} \varphi_{\mathrm{inc}}^{\mathrm{H}, \mathrm{PEO}}(Q, \omega)+\left(1-f_{\mathrm{PEO}}\right) \varphi_{\text {slow }}(Q, \omega)\right) .
$$

Basing on the experimental results of PMMA in the blend, as well as the simulation results, we can approximate $\varphi_{\text {slow }}(Q, \omega)$ by $\delta(\omega)$ for $T<400 \mathrm{~K}$ and at $400 \mathrm{~K}$, it will be the Fourier transform of a KWW function with $\beta=0.5$. Taking into account that no significant broadening can be observed in the low- $Q$ range, the analyses were performed in the range above $Q=0.4 \AA^{-1}$.

In a first approach, we choose the Fourier transform of a KWW function for $\varphi_{\text {inc }}^{\mathrm{H}, \mathrm{PEO}}(Q, \omega)$. At $300 \mathrm{~K}$, a fit with free $\beta$ parameter leads to a good description of the data with a $Q$-independent $\beta \approx 0.32$, and a reasonable small decrease of the associated DWF with $\left\langle u^{2}\right\rangle \approx 0.26 \AA^{2}$. For the stretching parameter $\beta$ a systematic tendency to increase with increasing temperature was revealed by the fits. As a maximum value we limited $\beta$ to that of pure PEO $(\beta=0.5)$ at $400 \mathrm{~K}$ and we interpolated linearly at intermediate temperatures (see Table II). In this way, we obtained satisfactory fits of the experimental data. For the scattering fraction $f_{\text {PEO }}$ we found values which at maximum deviated by $18 \%$ from its theoretical value of $f_{\mathrm{PEO}}=I_{\mathrm{inc}}^{\mathrm{H}, \mathrm{PEO}} / I_{\mathrm{total}}$. The slight increase found for $f_{\mathrm{PEO}}$ at $T>300 \mathrm{~K}$ can be due to several reasons, a change of the structure factor, different temperature dependencies for the DWF's, or possibly small quasielastic contributions of the coherent scattering could be accounted for by the fast PEO component in the fitting procedure. Considering the last possibility as rather likely, at $400 \mathrm{~K}$ we fixed $f_{\mathrm{PEO}}$ to its theoretical value, assuming that the Fourier transform of the KWW function in $\widetilde{S}_{\text {slow }}(Q, \omega)$ fully accounts for such a quasielastic contribution. We point out that, with this treatment, the DWF shows the proper $Q$ dependence. The average characteristic times, $\langle\tau\rangle=\tau_{w} \Gamma(1 / \beta) / \beta$, obtained for the fast and also for the slow component at $400 \mathrm{~K}$ are displayed as a function of $Q$ in Fig. 13. It can be observed that the relaxation times corresponding to $\mathrm{PEO}$ in the blend increase with decreasing temperature and that the time scale of this component is located in between those of pure PEO and PMMA in the blend at $400 \mathrm{~K}$. For the slow component, we note the excellent agreement with the relaxation times obtained from the analysis of PMMA in the blend; this finding strongly supports the consistency of our treatment at $400 \mathrm{~K}$. Concerning the $Q$ dependence, it is interesting to remark that the relaxation times related to the fast PEO component have some tendency to flatten at high- $Q$ values.

Now let us focus on the low- $Q$ spectra $\left(Q \leqslant 0.32 \AA^{-1}\right)$. Even at $T=400 \mathrm{~K}$, these spectra are elastic (see Fig. 14). On the other hand, if the PEO would undergo large scale relaxation (Rouse dynamics) we would expect also some quasielastic broadening at smaller $Q$. Taking Fig. 13 and comparing the $\tau$ values for pure PEO and PEO in the blend at $400 \mathrm{~K}$, we may estimate that at the smallest $Q$ 's, where both data reveal quasielastic signals, the difference is about one order of magnitude in time. According to de Gennes [62] the quasielastic relaxation time for the Rouse relaxation of the pair correlation function is given by

$$
\tau_{\mathrm{coh}}^{R}=\frac{36}{W \ell^{4}} Q^{-4},
$$

while that for the self-correlation function is given by Eq. (14). Using the result for pure PEO at $400 \mathrm{~K}$, where we found $W \ell^{4}=14200 \AA^{4} / \mathrm{ns}$, we would predict a relaxation time for the pair correlation function at $Q=0.32 \AA^{-1}$ of $\tau_{\text {coh }}$ $=0.24 \mathrm{~ns}$. For PEO in the blend the dynamics is at most one order of magnitude slower. From that we estimate $\tau_{\text {coh }}$ $\approx 2.4$ ns. For the next smallest $Q=0.24 \AA^{-1}$ this estimate becomes $\tau_{\text {coh }}\left(Q=0.24 \AA^{-1}\right)=7.6 \mathrm{~ns}$. Similarly the correlation times for the self-correlation function may be estimated to $\tau_{\text {inc }}\left(Q=0.32 \AA^{-1}\right)=1.9$ ns and $\tau_{\text {inc }}\left(Q=0.24 \AA^{-1}\right)=6.0$ ns. Us-

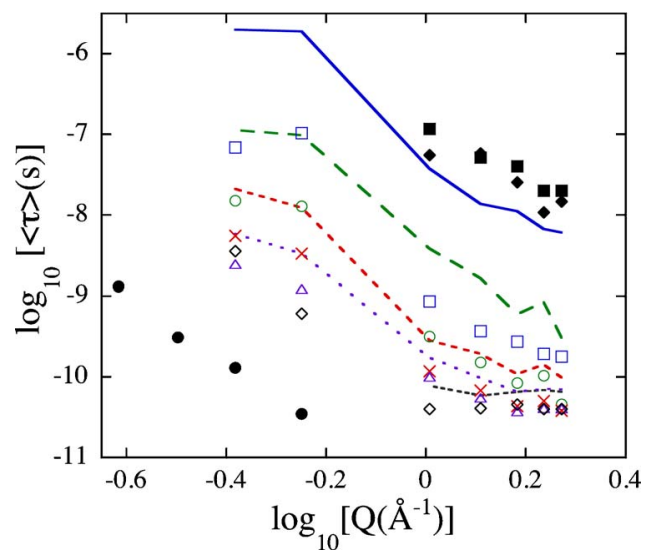

FIG. 13. (Color online) $Q$ dependence of the average time, $\langle\tau\rangle$, obtained from the fit of hPEO/dPMMA by a KWW and an elastic contribution at $300 \mathrm{~K}$ (squares), $325 \mathrm{~K}$ (circles), $350 \mathrm{~K}$ (crosses), $375 \mathrm{~K}$ (triangles) and two KWW functions at $400 \mathrm{~K}$ (diamonds; empty, fast component; full, slow component). The corresponding results when a log-normal distribution is used for the fast component are shown by the lines. Full squares represent the characteristic time for segmental dynamics of PMMA in the blend at $400 \mathrm{~K}$ obtained from the fit of the dPEO/hPMMA spectra (see Sec. V B 2). Full circles show the values obtained for $\langle\tau\rangle$ for pure PEO at $400 \mathrm{~K}$. 


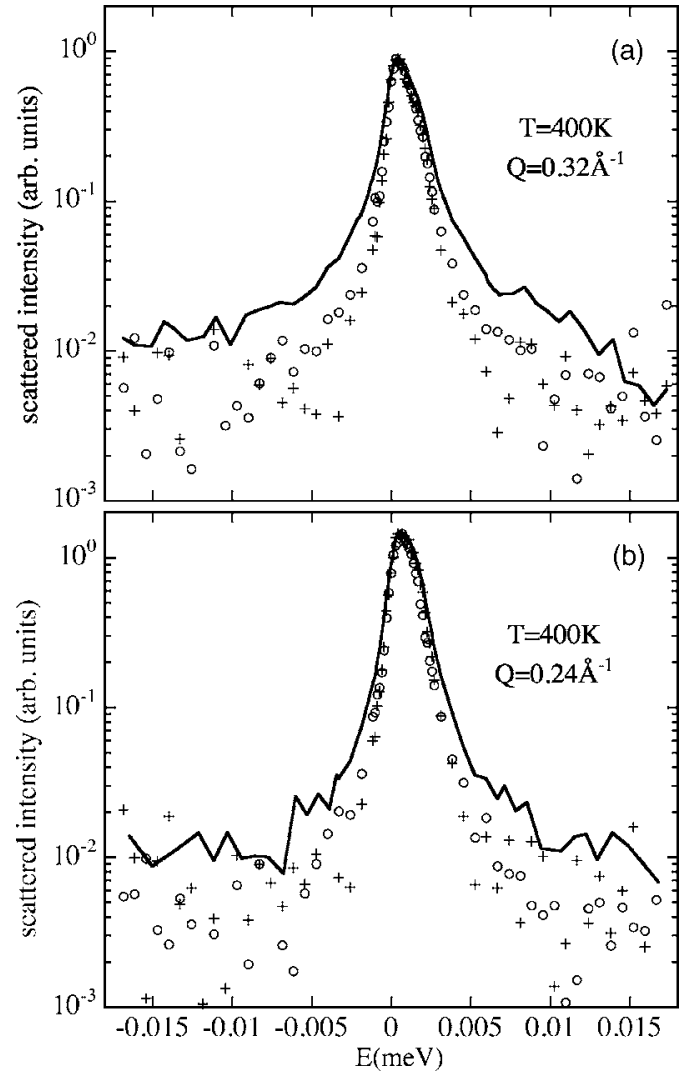

FIG. 14. Comparison of the observed neutron scattering spectra from PEO in the blend at $400 \mathrm{~K}$ (circles) at (a) $0.32 \AA^{-1}$ and (b) $0.24 \AA^{-1}$ to what would be predicted in terms of the Rouse model (solid lines). The plus signs show the instrumental resolution obtained at $5 \mathrm{~K}$.

ing the results obtained from the polarization analysis at $Q$ $=0.32 \AA^{-1}$ and $Q=0.24 \AA^{-1}$ the pair correlation function contributes to $65 \%$ and $75 \%$, respectively, to the observed spectra. With this information we may predict the Rouse relaxation function at the different $Q$ values. They are included in Fig. 14 and very clearly for both momentum transfers a significant broadening of the quasielastic spectra would be predicted.

Obviously, the dynamics observed at higher momentum transfers does not persist towards low $Q$. Thus, it must be spatially confined by the more or less rigid PMMA structure.

Seeking for further evidences of localization for PEO in the blend, we have scrutinized the self-part of the van Hove correlation functions, $G_{\text {self }}(r, t)$ [Eq. (1)], obtained from our MD trajectories of PEO hydrogens at both $400 \mathrm{~K}$ and $580 \mathrm{~K}$. These are displayed in Fig. 15 for different times between 5 ps and $16 \mathrm{~ns}$. At high temperature $(580 \mathrm{~K})$, the radial selfcorrelation function $4 \pi r^{2} G_{\text {self }}(r, t)$ shows [see Fig. 15(a)] the typical behavior previously obtained for different homopolymers above the glass-transition temperature (see, for instance, Ref. [44]). As time evolves, this function broadens and the maximum moves towards higher values of $r$. This is just the behavior expected for diffusivelike dynamics. However, at $400 \mathrm{~K}$, the behavior of $4 \pi r^{2} G_{\text {self }}(r, t)$ is qualitatively very different, in particular at longer times $(t \gtrsim 1 \mathrm{~ns})$ where a double peak structure develops [see Fig.15(b); note that the
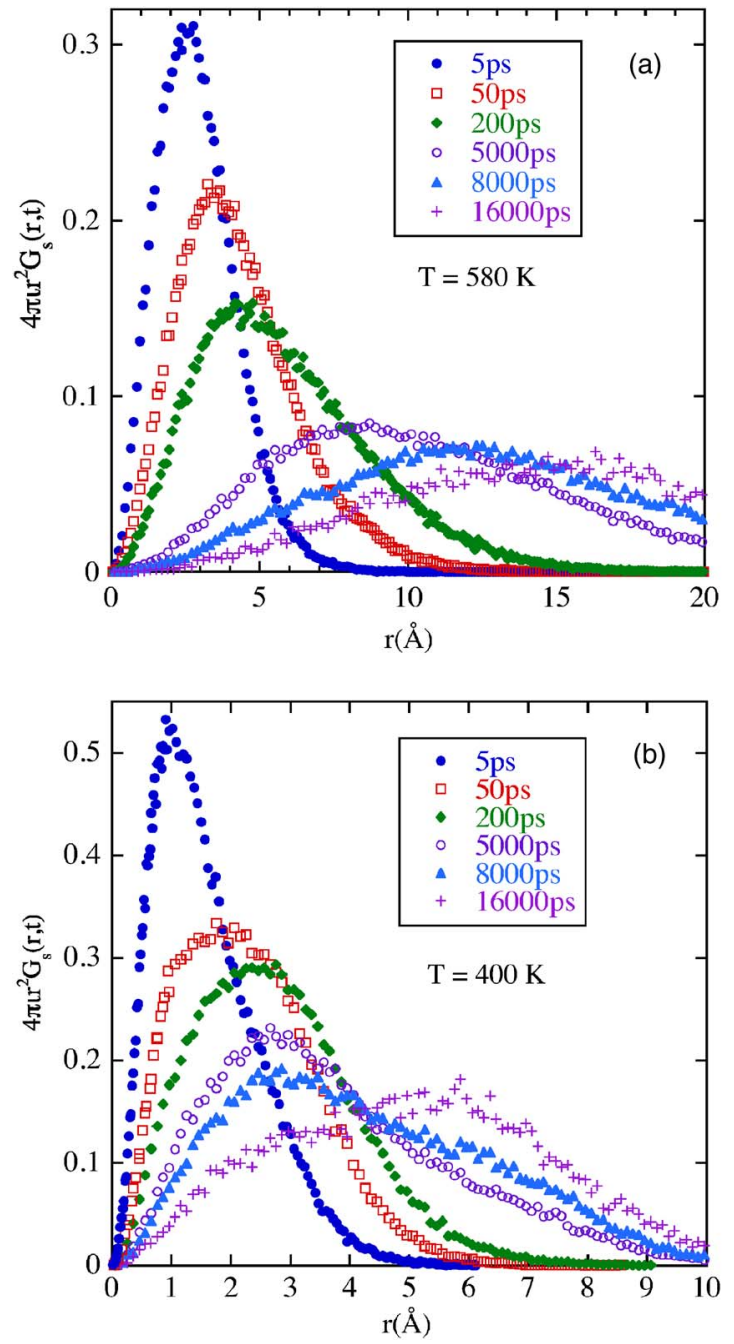

FIG. 15. (Color online) Radial self-correlation function for PEO hydrogens in the blend calculated from the MD simulations at 580 $\mathrm{K}$ (a) and $400 \mathrm{~K}$ (b) for the different times indicated.

scale of the $x$ axis is different from that in Fig.15(a)]. This could in principle indicate a heterogeneous-two populations-behavior. Moreover, the second maximum hardly moves with time between $1 \mathrm{~ns}$ and $16 \mathrm{~ns}$, suggesting that the atomic motions are localized, at least in this time regime. From the position of such a peak we can estimate a distance for these localized motions in the range of $6 \AA$ to 8 $\AA$. These findings strongly support again the idea that this localized motion regime is a consequence of some localization of the PEO chains created by the PMMA matrix (a real cage effect). This is in agreement with the results obtained by the Rouse analysis of the neutron measurements on the real sample. The cage effect will likely break only on a time scale where the PMMA matrix starts to relax.

Analysis in terms of distribution functions: The observation of elastic spectra at small $Q$ and $400 \mathrm{~K}$, as well as the MD-simulation results, strongly suggest the confined character of the PEO dynamics in the blend. To account for this, it is more appropriate to approximate the fast component in Eq. (20) $\left[\varphi_{\text {inc }}^{\mathrm{H}} \mathrm{PEO}(Q, \omega)\right]$ as a log-normal distribution of relaxation times, 


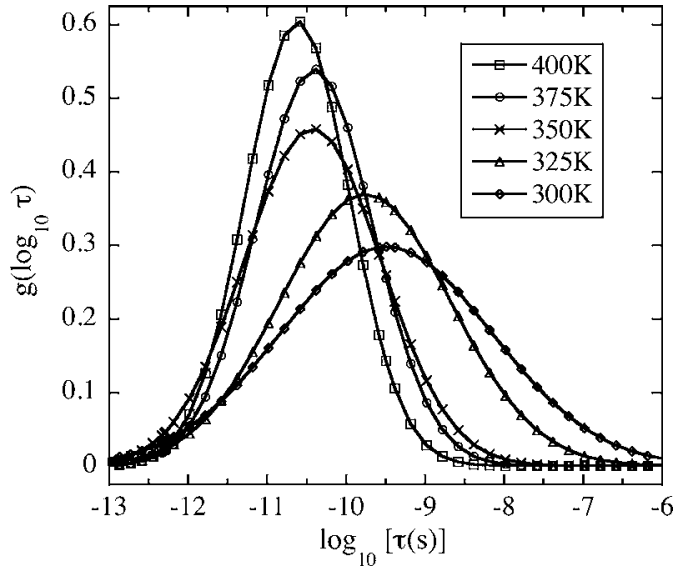

FIG. 16. Temperature dependence at $Q=1.02 \AA^{-1}$ of the lognormal distribution obtained for PEO in the blend at the temperatures indicated.

$$
\begin{aligned}
\varphi_{\text {inc }}^{\mathrm{H}, \mathrm{PEO}}(Q, \omega)= & \int_{-\infty}^{+\infty} \frac{1}{\sqrt{2 \pi} \sigma} \exp \left(-\frac{\left(\ln \tau-\ln \tau_{0}\right)^{2}}{2 \sigma^{2}}\right) \\
& \times \frac{1}{\pi} \frac{\tau}{1+\omega^{2} \tau^{2}} d(\ln \tau)
\end{aligned}
$$

(the distributed Lorentzian functions are the Fourier transforms of the exponential decays in the time domain). The

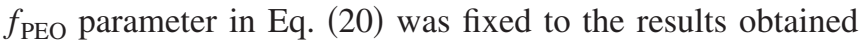
from the previous analysis in terms of stretched exponentials. In the case of $400 \mathrm{~K}$, the quasielastic broadening of the slow component was fixed to the values independently obtained from the analysis of the dPEO/hPMMA sample. As we observed that the width parameters $\sigma$ did not depend significantly on $Q$, they were kept constant to the average values obtained for each temperature (see Table II). The solid lines in Fig. 5 show some representative fitting curves at $Q$ $=1.02 \AA^{-1}$ and different temperatures and we note the good description of the experimental data. Again, it is worthy of remark that the DWF follows a consistent evolution from $300 \mathrm{~K}$ to $400 \mathrm{~K}$, leading to the values for $\left\langle u^{2}\right\rangle$ shown in Table II. In Fig. 13, we have plotted the resulting characteristic times, compared with the results obtained from our first approach. Because of the large uncertainties for the fits at low$Q$ values at $400 \mathrm{~K}$, the corresponding times have been omitted. It is noteworthy that the use of a log-normal distribution for the fast PEO component, instead of one KWW function, leads to time scales shifted towards slower times. The temperature dependent distribution functions obtained at $Q$ $=1.02 \AA^{-1}$ are shown in Fig. 16 .

\section{DISCUSSION}

\section{A. Pure PEO}

As we have shown in the analysis section (Fig. 12), the characteristic times observed from our pure PEO sample over the entire $Q$ regime follows well the $\tau_{w} \propto Q^{-4}$ power law associated with the entropy driven Rouse dynamics of polymer chains in the universal regime, where topological con- straints are not yet active. We note that, other than for most polymers [50], the Rouse regime appears to be valid up to $Q$ values of $Q \approx 0.6 \AA^{-1}$, indicating very flexible chains. For many polymers the Rouse $Q^{-4}$ power law is only observed for $Q \leqslant 0.2 \AA^{-1}$ [e.g., polyisobutylene (PIB), poly(vinyl ethylene) (PVE), polyisoprene (PI), etc.] [47,50,63-65]. Beyond that, the so-called intrachain viscosity effects limit the Rouse behavior towards smaller length scales [63]. These are dissipative processes related, e.g., to rotational transition effects inducing deviations from the universal $Q^{-4}$ regime. The observation of a Rouse regime extending up to $Q \approx 0.6 \AA^{-1}$ sets PEO in the same rows as poly(dimethyl siloxane) (PDMS), where also very little intrachain friction was found [64]. The value of the characteristic Rouse relaxation rate $W \ell^{4}(400 \mathrm{~K}) \approx 14200 \AA^{4} / \mathrm{ns}$ compares well with other highly mobile polymers like polyethylene-propylene (PEP), $W \ell^{4}(423 \mathrm{~K}) \approx 9500 \AA^{4} / \mathrm{ns}$ or polyethylene (PE), where $W \ell^{4}(418 \mathrm{~K}) \approx 14700 \AA^{4} / \mathrm{ns}$ holds [50]. Only PDMS appears to be more mobile with $W \ell^{4}(373 \mathrm{~K}) \approx 18000 \AA^{4} / \mathrm{ns}$.

Figure 12(b) displays the temperature dependence of the characteristic relaxation rate in relation to other findings. First, we note that earlier neutron spin-echo experiments on the single chain dynamic structure factor [59] reveal very similar Rouse relaxation rates as the present experiments. The agreement between results obtained from different correlators and with different instruments supports the application here made of the Rouse model to the BSS data. Furthermore, ${ }^{1} \mathrm{H}$ NMR experiments reported by Cohen-Addad et al. [7] lead to very similar relaxation rates, if we compare them with the extrapolated neutron data corresponding to $Q$ $\leqslant 1 \AA^{-1}$. On the other hand, the recently published deuterium NMR data by Lutz et al. [20] appear to display a weaker temperature dependence and are also significantly slower.

\section{B. PEO in the blend with PMMA}

The single most important finding of this work is the direct observation of very heterogeneous confined dynamics of the low- $T_{g}$ material in the high- $T_{g}$ blend matrix. Before we allude to the significance of this observation we first like to go through the different pieces of information which led to this picture. We started our evaluation with the standard approach used in glass-forming polymers, namely with a description of the observed spectra in terms of KWW functions. These fits resulted in very small stretching parameters in particular at low temperatures $(\beta \approx 0.3)$ which increased with increasing temperature indicating very broad distributions of the relaxation times. In glass-forming polymers in general the shape of the relaxation function characterized by the stretching exponent $\beta$ is reflected in the $Q$ dispersion of the relaxation times, $\tau_{w} \propto Q^{-2 / \beta}[66,67]$. This law signifies the validity of the Gaussian approximation and can be interpreted in terms of an anomalous diffusion process as the mechanism behind the $\alpha$ process. For PEO in PMMA this shape/dispersion relation is grossly invalidated, indicating the non-Gaussianity or heterogeneity of the system.

The dynamics, however, is not only heterogeneous but moreover confined in space. This was revealed from the 


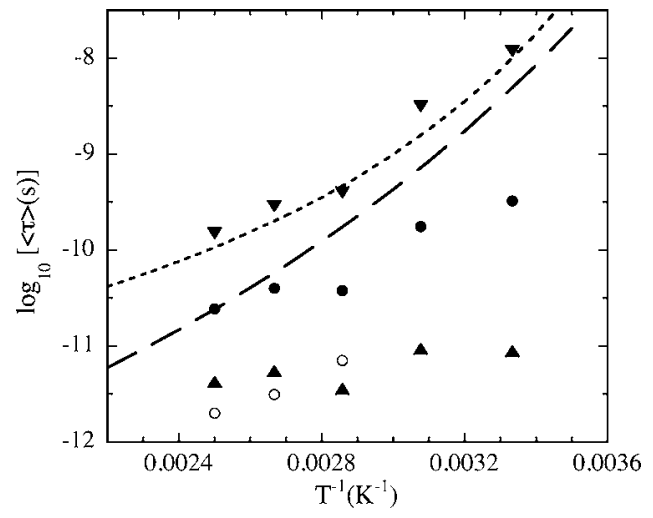

FIG. 17. Temperature dependence of the average relaxation time obtained from this work for PEO pure (empty circles) for $Q$ $=1.02 \AA^{-1}$. The triangles show the evolution of the slow and fast side of the log-normal distribution functions shown in Fig. 16 and the full circles display $\tau_{0}$. The results obtained from NMR measurements are displayed for comparison (dashed line, Ref. [7]; dotted line, Ref. [20]).

missing broadening of these spectra measured at small $Q$ values $\left(Q \leqslant 0.32 \AA^{-1}\right)$. Even a very conservative estimation of the quasielastic linewidth which is to be expected from an extrapolation of the higher- $Q$ data (Fig. 14) would have predicted significant broadenings of the low- $Q$ spectra, which were not observed. Thus, the presence of the hardly moving PMMA matrix, which below $335 \mathrm{~K}$ - the average $T_{g}$ of the blend-is completely frozen, leads to the creation of little pockets of mobility where the PEO can move. From the $Q$ value, where this effect appears $\left(Q \leqslant 0.3 \AA^{-1}\right)$ a characteristic size of these mobility pockets of about $\ell_{\text {conf }} \approx \pi / Q$ $\approx 1 \mathrm{~nm}$ may be estimated. We note that a real space evaluation of the computer simulation data suggests qualitatively a similar scenario.

The confined character of the motion together with the non-Gaussianity of the relaxation function suggested the description of the spectra in terms of log-normal distributions instead of stretched exponentials. The excellent results of this approach (see Fig. 5) underlines the appropriateness of this approach.

As can be seen in Fig. 16, the resulting distribution functions shift with increasing temperatures to shorter times and at the same time become narrower. It is interesting to note that the flank corresponding to the slower times of this distribution is much more strongly shifting with temperature than the shorter times counter part. Obviously, there appear to be PEO segments which are very much less affected by the confinement than others which need to be much more activated in order to move. Figure 17 displays the temperature dependence of the characteristic times corresponding to these distributions. The full circles characterize the average distribution times, $\tau_{0}$, while the upper and lower triangles display the times connected with the slow and fast side of the log-normal distribution functions shown in Fig. 16. The dotted line displays the result from the ${ }^{2} \mathrm{H}$ NMR [20] while the dashed line gives the ${ }^{1} \mathrm{H}$ NMR results [7]. Finally, the open circles present the average relaxation times obtained from pure PEO. The comparisons of Fig. 17 are revealing. The

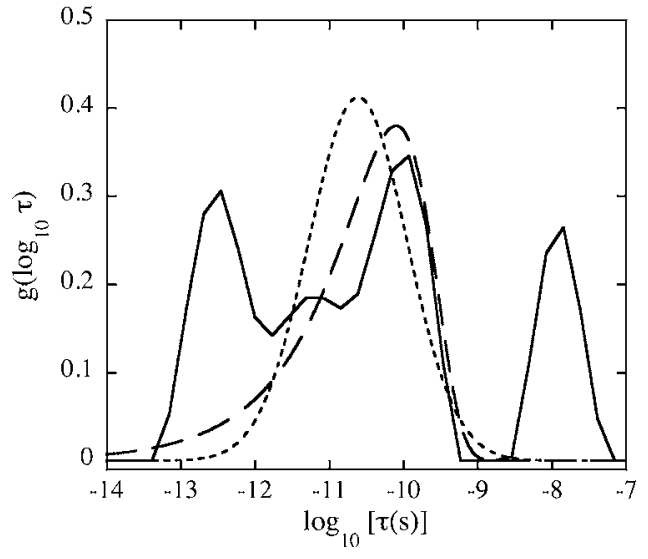

FIG. 18. Distribution of relaxation times underlying the PEO dynamics in the blend as obtained from the MD simulations by inverse Laplace transformation (solid line) and from the experimental spectra by using a log-normal distribution (dotted line) or a stretched exponential (dashed line). Data correspond to $Q$ $=1.02 \AA^{-1}$ and $400 \mathrm{~K}$.

short-time flank of the distribution functions appears to relate to those PEO segments, which are embedded in an environment very similar to that of pure PEO. Here the dynamics is nearly quantitatively identical. On the other hand, the very good agreement of the ${ }^{2} \mathrm{H}$ NMR results with those from the long time flank of the distribution function indicates that this technique appears to be most sensitive to the slowly moving deuteriums, an observation which in a similar way holds also for the ${ }^{1} \mathrm{H}$ NMR data. The fast moving segments apparently are subsumed in the amplitude factors which in the NMR studies were related to librational and other types of fast dynamics.

We now compare the experimentally determined relaxation time distribution functions with the outcome of the MD simulations. For that purpose we have performed an inverse Laplace transformation (ILT) of the simulated spectra (CONTIN analysis, see e.g., Ref. [68] and references therein). Figure 18 displays the results for $Q=1 \AA^{-1}$. Three different regimes are revealed. At short times $(\tau<1 \mathrm{ps})$, a narrow peak evolves relating to the librational and vibrational dynamics which occurs in the subpicosecond regime. We note that this peak is not moving with $Q$. Second, in the time regime between nanoseconds and picoseconds a broad double peak is observed relating to the segmental relaxation processes. Finally, at even longer times some further structure appears which is related to the break of the "confinement effect" (plateau) shown by the $S_{\text {inc }}(Q, t)$ at the edge of the simulation time. Due to this reason, this breaking (and thereby the longest-times peak of ILT) needs to be confirmed by longer simulations. Figure 18 also includes the fitted lognormal distribution function for the segmental relaxation times as obtained from the data fit of a spectrum at $T$ $=400 \mathrm{~K}$ and $Q=1 \AA^{-1}$. Furthermore, a corresponding distribution function relating to a stretched exponential obtained from the same spectrum is also shown. Both, concerning its position as well as its width, the experimental distribution function agrees very well with the simulated time distribution function. On the other hand, the distribution function 


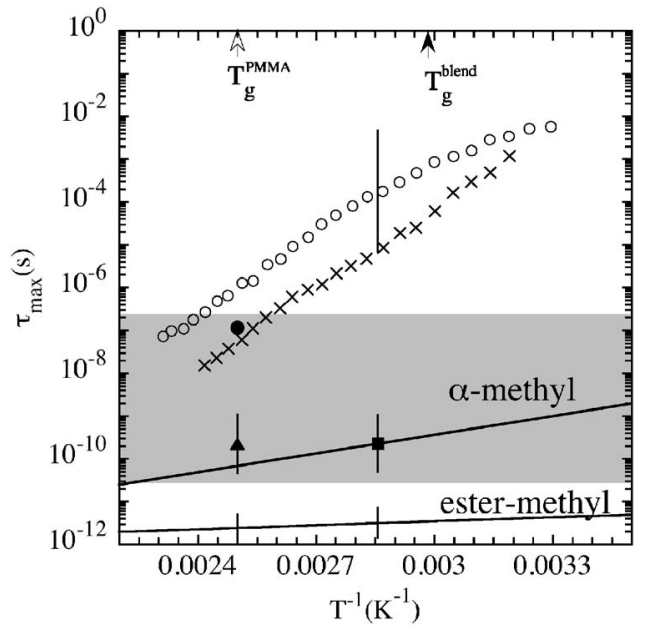

FIG. 19. Arrhenius plot for the position of the maximum of the distribution of relaxation times $\tau_{\max }$ for PMMA in the blend obtained in this work, $\alpha$-methyl rotation (full square, BSS; full triangle, MD simulations) and additional slower relaxation at $Q$ $=1 \AA^{-1}$ (full circle, BSS). They are compared with data from the literature, inverse of the dielectric loss maxima (open circles, pure PMMA; crosses, 25\% PEO/75\% PMMA) [13] and ester-methyl rotation for PMMA (solid line on the bottom) [48]. The widths of the associated distributions of relaxation times (half-maximum) are indicated by the vertical lines (for the dielectric spectra of pure PMMA at $350 \mathrm{~K}$ the data are taken from Ref. [69]). The shadowed area indicates the time scales accessible with BSS.

corresponding to the stretched exponential relaxation develops a tail well into the regime of microscopic times, which is unphysical. By construction, the experimental data that were obtained by a fit of one log-normal distribution function cannot reveal the double peak structures seen from the simulations. Given the narrow experimental window, such an observation could also not have been expected. However, the different temperature dependences observed for the two flanks of the log-normal distribution could be a signature of such an underlying double structure.

\section{PMMA in the blend with PEO}

At the temperatures investigated, the most pronounced feature in the dPEO/hPMMA spectra in the mesoscopic time scale covered by BSS is the $\alpha$-methyl rotation. The agreement found for the parameters characterizing this dynamics (time scales as well as the width of the distribution functions) obtained from the experimental and the MDsimulations data is beautiful, even taking into account that the concentration of PEO in the blend is different for the simulated and the experimental sample (see Fig. 19).

Concerning the additional dynamics superimposed at 400 $\mathrm{K}$, we first note that the time scales found for main-chain and $\alpha$-methyl hydrogens from the simulations are very close to each other, strongly supporting the assumption of statistically independent motions for $\alpha$-methyl hydrogens. The estermethyl hydrogen slow dynamics is also rather close to the main-chain motions (only a factor 4 is found between both time scales). This indicates a strong coupling of the side- group dynamics with the overall segmental dynamics. The slightly faster motion of the ester-methyl hydrogens might reflect that the side group is also involved in other additional motion as, e.g., those leading to the pronounced $\beta$ relaxation in this polymer [69]. A higher mobility of the hydrogens of this methyl group is also found in the microscopic region, as reflected by the higher value obtained for $\left\langle u^{2}\right\rangle$. Now, considering the experimental results, a shift in the absolute scale with respect to the MD-simulations results is observed, that might be due to the different compositions of the real and simulated samples. On the other hand, the power laws obtained for the characteristic times are very similar in all cases and reveal much weaker $Q$ dependencies than that characteristic for Gaussian behavior $\left(\tau_{w} \propto Q^{-2 / \beta}\right)$. As commented above, this can be considered as a natural consequence of the heterogeneous character of the blend.

We note here again the excellent agreement found for the time scale associated to the additional slow dynamics as obtained from the independent analysis of the dPEO/hPMMA and hPEO/dPMMA samples. This coincidence is nontrivial and evidences the consistency of the overall approach followed in this work. On the other hand, in Fig. 19 we compare the value for this time scale at $Q=1 \AA^{-1}$ with dielectric results on the same blend [13]. Also in this case, as usually observed in glass-forming polymers [70] at this $Q$ value the incoherent relaxation times match the relaxation results. At $400 \mathrm{~K}$ ( $\approx 65 \mathrm{~K}$ above the average $T_{g}$ of the blend) the dielectric spectrum reflects what is usually called the merged $\alpha \beta$ process, and this is seemingly also the process revealed by our neutron scattering spectra. Finally, we note that at $350 \mathrm{~K}$ the maximum of the dielectric losses is still located at sufficiently slow times to be observed by BSS, even taking into account the width of this kind of processes (see as reference in Fig. 19 the width reported for pure PMMA at $350 \mathrm{~K}$ in Ref. [69]). It is worth also remarking about the huge difference found for the mobility of the two components in the blend in the high- $Q$ range where both could be characterized $\left(Q \geqslant 1 \AA^{-1}\right)$, more than 3 orders of magnitude separate the time scales for PEO and PMMA in the blend at $400 \mathrm{~K}$.

We finally comment on the comparison of our results with those published by Sakai et al. [21] on a similar blend (dPEO/hPMMA with $20 \%$ of PEO). In that work the backscattering data obtained in the $T$ range $308 \mathrm{~K}-490 \mathrm{~K}$ were Fourier transformed to the time domain and analyzed in terms of a single KWW function. At $Q \approx 1 \AA^{-1}$ the time scale deduced for $405 \mathrm{~K}$ is rather close to the value we obtain for the segmental motion of PMMA. However, the $Q$ dependence obtained is weaker $\left(\approx Q^{-2}\right.$ from that work). This can be due to the fact that they did not take into account the $\alpha$-methyl rotation in their data analysis procedure.

\section{On the role of computer simulations in the evaluation of data from complex systems}

Atomistic MD simulations reveal the time-dependent trajectories of all atoms in a sample and contain in principle the same information as a quasielastic or inelastic neutron scattering experiment which addresses the space-time correlation functions again connected to the trajectories of all atoms of a 
sample. While it is also possible in a scattering experiment to highlight certain classes of atoms by partial hydrogenation or deuteration, in a simulation the contribution of each class of atoms can be directly evaluated. Furthermore, a real space analysis of the van Hove correlation functions may permit direct evidence on the spatial extent of motion. In a complex system with many different atoms, MD simulations therefore can be an invaluable tool, in order to disentangle the complex experimental spectra which always contain contributions from many different types of atoms.

In this work the simulations corroborated the key experimental observations, (i) the confined nature and heterogeneity of the dynamics of the PEO component; (ii) it allowed to identify the PMMA dynamical contributions to the central component in the hPEO/dPMMA spectra; (iii) it revealed the predominance of the $\alpha$-methyl group motion for the PMMA dynamics, and (iv) it also showed very well the nonappropriateness of stretched exponentials for the characterization of the PEO dynamics; (v) finally, due to their much larger dynamic range the simulations strongly supported and assured the interpretation of the experimental results which unavoidably were based on a small experimental observation window. Thus, in the study of complex dynamics, MD simulations facilitate a much more profound and deep data interpretation than what would be possible without their support. As recent examples on this potential we can mention, (i) the identification of the finite jump length associated to the subdiffusive motion in the $\alpha$ relaxation as the source of deviations from Gaussian behavior in homopolymers [52,71] and (ii) the unraveling of dynamic heterogeneities inherent to the chemical structure in a polymer with side groups as PVE [72].

\section{E. What did we learn?}

We have shown that the blending of two miscible polymers with very different glass-transition temperatures leads to a complete decoupling of the local segmental dynamics of the fast component from that of the low $T_{g}$ matrix. The phenomenon occurs on a length scale of about $1 \mathrm{~nm}$ and may be interpreted in terms of small PEO enclosures within the still frozen or very little mobile PMMA matrix. Certainly this length scale may vary with concentration of the fast component. Beyond this length scale the large scale dynamics of the fast component is strongly slowed down. This is both revealed by the experimental low- $Q$ spectra, as well as by the real space evaluation of the simulations.

We suggest that the dynamical decoupling between the low and high $T_{g}$ component in the neighborhood of the average glass-transition temperature of the blend including confinement effects in terms of mobility pockets is a more general phenomenon. If, in a miscible blend, the two component glass-transition temperatures are different enough, at the glass-transition temperature of the blend the overall matrix freezes but the faster component is still active. In this situation, for the dynamical behavior of the low- $T_{g}$ component, we could expect a crossover from a glass-forming liquidlike behavior at high temperature towards some kind of confined behavior at low temperature. This crossover should take place around the glass-transition temperature of the blend, and should manifest as a deviation from the high temperature $\tau(T)$ dependence (non-Arrhenius) towards some kind of Arrhenius-type behavior (signature of confinement) at low temperatures. In fact, this kind of crossover was already observed by dielectric spectroscopy for the dynamics of PVME in blends with PS and with high concentration of PS [19]. In that presentation the observed crossover was interpreted in terms of confinement effects and a confinement length of about $1 \mathrm{~nm}$ was suggested. Similar dielectric results have been obtained in other systems as PVME/ poly(o-chlorostyrene) and PS/poly(phenylene oxide) [73].

For PEO/PMMA, because of the extremely large $\Delta T_{g}$, the difference in segmental dynamics around the blend glass transition temperature is particularly large and therefore, its observation came into the window of neutron scattering techniques allowing a space-time scrutiny of the underlying dynamics. Similarly also computer simulations were feasible. A direct evidence of confinement is thereby obtained by these methods.

Although confinement effects are more evident for blends with a high concentration of the high- $T_{g}$ component, they could also affect the dynamics of the low- $T_{g}$ component in blends with different concentrations and large $\Delta T_{g}$. The reason is that due to concentration fluctuations, some regions within the blend can experience very high local concentrations of the high- $T_{g}$ component. Therefore, even when the bulk concentration of the high- $T_{g}$ component is not very high, the dynamics of the low- $T_{g}$ component could deviate from the expected liquidlike equilibrium behavior as the temperature decreases towards the average glass transition of the blend. This may be one of the reasons of the very large differences in the segmental dynamics at low temperature in large $\Delta T_{g}$ miscible blends. For instance, in the $50 \% / 50 \%$ $\mathrm{PI} / \mathrm{PVE}$ blend close to the glass-transition temperature of the blend of $T_{g} \approx 228 \mathrm{~K}$ the component dynamics differ by more than 3 orders of magnitude. It is worthy of remark that these nonequilibrium effects are not taken into account by any of the existing models for blend dynamics.

\section{ACKNOWLEDGMENTS}

The authors thank Dr. Hans Grimm and Dr. Werner Schweika for their help at the BSS and DNS instruments, respectively. This research project has been supported by the European Commission, NMI3 Contract No. RII3-CT-2003505925 and the NoE SoftComp, Contract No. NMP3-CT2004-502235. Three of the authors (A. A., J. C., F. A.) acknowledge support from the Project MAT2004-01017. Support from "Donostia International Physics Center" is also acknowledged. 
[1] C. Brosseau, A. Guillermo, and J. P. Cohen-Addad, Macromolecules 25, 4535 (1992).

[2] G.-C. Chung, J. A. Kornfield, and S. D. Smith, Macromolecules 27, 5729 (1994).

[3] B. H. Arendt, R. M. Kannan, M. Zewail, J. A. Kornfield, and S. D. Smith, Rheol. Acta 33, 322 (1994).

[4] A. Alegría, C. Elizetxea, I. Cendoya, and J. Colmenero, Macromolecules 28, 8819 (1995).

[5] F. Alvarez, A. Alegría, and J. Colmenero, Macromolecules 30, 597 (1997).

[6] B. H. Arendt, R. Krishnamoorti, J. A. Kornfield, and S. D. Smith, Macromolecules 30, 1127 (1997)

[7] C. Lartigue, A. Guillermo, and J. P. Cohen-Addad, J. Polym. Sci., Part B: Polym. Phys. 35, 1095 (1997).

[8] A. Guillermo, C. Lartigue, and J. P. Cohen-Addad, Macromolecules 31, 769 (1998).

[9] I. Cendoya, A. Alegría, J. M. Alberdi, J. Colmenero, H. Grimm, D. Richter, and B. Frick, Macromolecules 32, 4065 (1999).

[10] A. Arbe, A. Alegría, J. Colmenero, S. Hoffmann, L. Willner, and D. Richter, Macromolecules 32, 7572 (1999).

[11] S. Adams and D. B. Adolf, Macromolecules 32, 3136 (1999).

[12] S. Hoffmann, L. Willner, D. Richter, A. Arbe, J. Colmenero, and B. Farago, Phys. Rev. Lett. 85, 772 (2000).

[13] M. Dionísio, A. C. Fernandes, J. F. Mano, N. T. Correia, and R. C. Sousa, Macromolecules 33, 1002 (2000).

[14] M. Doxastakis, M. Kitsiou, G. Fytas, D. N. Theodorou, N. Hadjichristidis, G. Meier, and B. Frick, J. Chem. Phys. 112, 8687 (2000).

[15] X. Yang, A. Halasa, W.-L. Hsu, and S.-Q. Wang, Macromolecules 34, 8532 (2001).

[16] B. Min, X. Qiu, M. D. Ediger, M. Pitsikalis, and N. Hadjichristidis, Macromolecules 34, 4466 (2001).

[17] S. H. Zhang, P. C. Painter, and J. Runt, Macromolecules 35, 9403 (2002).

[18] M. Doxastakis, K. Chrissopoulou, A. Aouadi, B. Frick, T. P. Lodge, and G. Fytas, J. Chem. Phys. 116, 4707 (2002).

[19] C. Lorthioir, A. Alegría, and J. Colmenero, Phys. Rev. E 68, 031805 (2003)

[20] T. R. Lutz, Y. He, M. D. Ediger, H. Cao, G. Lin, and A. A. Jones, Macromolecules 36, 1724 (2003).

[21] V. G. Sakai, C. Chen, J. K. Maranas, and Z. Chowdhuri, Macromolecules 37, 9975 (2004).

[22] R. Mukhopadhyay, A. Alegría, J. Colmenero, and B. Frick, J. Non-Cryst. Solids 235-237, 233 (1998).

[23] A. Zetsche and E. W. Fischer, Acta Polym. 45, 168 (1994).

[24] G. Katana, E. W. Fischer, T. Hack, V. Abetz, and F. Kremer, Macromolecules 28, 2714 (1995)

[25] E. Donth, J. Non-Cryst. Solids 53, 325 (1982).

[26] E. Donth, J. Non-Cryst. Solids 131, 204 (1991).

[27] E. Donth, The Glass Transition. Relaxation Dynamics in Liquids, Disordered Materials (Springer, Berlin, 2001).

[28] S. K. Kumar, R. H. Colby, S. H. Anastasiadis, and G. Fytas, J. Chem. Phys. 105, 3777 (1996).

[29] T. P. Lodge and T. C. B. McLeish, Macromolecules 33, 5278 (2000).

[30] B. E. Read, Polymer 3, 529 (1962).

[31] W. B. Wu, W. Y. Chiu, and W. B. Liau, J. Appl. Polym. Sci. 64, 411 (1997).

[32] T. Springer, Quasielastic Neutron Scattering for the Investiga- tion of Diffusive Motions in Solids, Liquids, Springer Tracts in Modern Physics (Springer-Verlag, Berlin, Heidelberg, New York, 1972), Vol. 64.

[33] S. W. Lovesey, Theory of Neutron Scattering from Condensed Matter (Clarendon, Oxford, 1984).

[34] M. Bée, Quasielastic Neutron Scattering (Adam Hilger, Bristol, 1988).

[35] G. L. Squires, Introduction to the Theory of Thermal Neutron Scattering (Dover, New York, 1996).

[36] http://www.fz-juelich.de/iff/wns/

[37] B. Alefeld, M. Birr, and A. Heidemann, Naturwiss. 56, 410 (1969).

[38] H. Sun, Macromolecules 28, 701 (1995).

[39] H. Sun, S. J. Mumby, J. R. Maple, and A. T. Hagler, J. Phys. Chem. 99, 5873 (1995).

[40] H. Sun, J. Phys. Chem. B 102, 7338 (1998).

[41] H. Sun, S. J. Mumby, J. R. Maple, and A. T. Hagler, J. Phys. Chem. 102, 7338 (1998).

[42] D. N. Theodorou and U. W. Suter, Macromolecules 19, 139 (1986).

[43] D. N. Theodorou and U. W. Suter, Macromolecules 19, 379 (1986).

[44] J. Colmenero, F. Alvarez, and A. Arbe, Phys. Rev. E 65, 041804 (2002).

[45] B. Frick, D. Richter, and Cl. Ritter, Europhys. Lett. 9, 557 (1989).

[46] B. K. Annis, O. Borodin, G. D. Smith, C. J. Benmore, A. K. Soper, and J. D. Londono, J. Chem. Phys. 115, 10998 (2001).

[47] D. Richter, M. Monkenbusch, L. Willner, A. Arbe, J. Colmenero, and B. Farago, Europhys. Lett. 66, 239 (2004).

[48] A. J. Moreno, A. Alegría, J. Colmenero, and B. Frick, Macromolecules 34, 4886 (2001).

[49] M. P. Allen and D. J. Tildesley, Computer Simulation of Liquids (Oxford Science Publications, Clarendon Press, Oxford, 2001).

[50] D. Richter, M. Monkenbusch, A. Arbe, and J. Colmenero, Neutron Spin Echo Investigations on Polymer Dynamics, Advances in Polymer Science (Springer-Verlag, Berlin, Heidelberg, New York, 2005), Vol. 174.

[51] D. Richter, A. Arbe, J. Colmenero, M. Monkenbusch, B. Farago, and R. Faust, Macromolecules 31, 1133 (1998).

[52] A. Arbe, J. Colmenero, F. Alvarez, M. Monkenbusch, D. Richter, B. Farago, and B. Frick, Phys. Rev. E 67, 051802 (2003).

[53] R. Pérez, A. Arbe, J. Colmenero, B. Frick, L. Willner, and D. Richter (unpublished).

[54] F. Alvarez, A. Arbe, and J. Colmenero, Chem. Phys. 261, 47 (2000).

[55] A. Chahid, A. Alegría, and J. Colmenero, Macromolecules 27, 3282 (1994).

[56] R. Mukhopadhyay, A. Alegría, J. Colmenero, and B. Frick, Macromolecules 31, 3985 (1998).

[57] M. Doi and S. F. Edwards, The Theory of Polymer Dynamics (Clarendon, Oxford, 1986).

[58] P. E. Rouse, Jr., J. Chem. Phys. 21, 1272 (1953).

[59] A. Wischnewski et al. (private communication).

[60] G. Allen, C. J. Wright, and J. S. Higgins, Polymer 15, 319 (1974).

[61] A. J. Moreno, A. Alegría, and J. Colmenero (unpublished).

[62] P. G. de Gennes, Physics (Long Island City, N.Y.) 3, 37 (1967). 
[63] D. Richter, M. Monkenbusch, J. Allgaier, A. Arbe, J. Colmenero, B. Farago, Y. Cheol Bae, and R. Faust, J. Chem. Phys. 111, 6107 (1999).

[64] A. Arbe, M. Monkenbusch, J. Stellbrink, D. Richter, B. Farago, K. Almdal, and R. Faust, Macromolecules 34, 1281 (2001).

[65] A. Arbe, Physica B 350, 178 (2004).

[66] J. Colmenero, A. Alegría, A. Arbe, and B. Frick, Phys. Rev. Lett. 69, 478 (1992).

[67] A. Arbe, J. Colmenero, M. Monkenbusch, and D. Richter, Phys. Rev. Lett. 81, 590 (1998).

[68] A. Alvarez, A. Alegría, and J. Colmenero, J. Chem. Phys. 103,
798 (1995).

[69] R. Bergman, F. Alvarez, A. Alegría, and J. Colmenero, J. NonCryst. Solids 235-237, 580 (1998).

[70] J. Colmenero, A. Arbe, and A. Alegría, J. Non-Cryst. Solids 172-174, 126 (1994).

[71] A. Arbe, J. Colmenero, F. Alvarez, M. Monkenbusch, D. Richter, B. Farago, and B. Frick, Phys. Rev. Lett. 89, 245701 (2002).

[72] A. Narros, F. Alvarez, A. Arbe, J. Colmenero, D. Richter, and B. Farago, J. Chem. Phys. 121, 3282 (2004).

[73] D. Cangialosi, A. Alegría, and J. Colmenero (unpublished). 